\title{
卤素功能化靛红的合成及其烃基化、还原和酰基化反应研究
}

\author{
高文涛* 赵鹏波赵宾宾李阳
}

(渤海大学超精细化学品研究所 锦州 121000)

\begin{abstract}
摘要 报道了以 4-卤代苯胺为原料经 Sandmeyer 反应得到卤素功能化靛红 3a $\sim 3 \mathbf{c}$, 进一步烃基化反应得到氮上烃基化 产物 4a 40. 4a 40 用水合肼还原得到了吲哚酮产物 5a $\sim 50,5 a \sim 50$ 用乙酸䣫作酰化试剂在 4-二甲氨基吡啶为催化剂 的条件下反应生成 2,3 位酰基化的中间体，此中间体不经提纯，而使酯基在四氢呋喃为溶剂、5\%氢氧化钠为碱的条件 下水解得到 3 位酰基化产物 $\mathbf{6 a} \sim \mathbf{6 o}$. 部分化合物未见文献报道, 其结构经红外光谱、质谱、核磁氢谱(碳谱)和元素分析 确认.
\end{abstract}

关键词 卤素功能化靛红; 还原; 烃基化; 乙酰化

\section{Synthesis, Alkylation, Reduction and Acylation of Halo-functionalized Isatins}

\author{
Gao, Wentao* Zhao, Pengbo Zhao, Binbin Li, Yang \\ (Institute of Superfine Chemicals, Bohai University, Jinzhou 121000)
}

\begin{abstract}
In this paper, 4-haloanilines were first underwent the Sandmeyer reaction to give the corresponding halo-functionalized isatins $\mathbf{3 a} \sim \mathbf{3 c}$, which were further alkylated at the nitrogen atom to give $\mathbf{4 a} \sim \mathbf{4 0}$ followed by in situ reduction using hydrazine hydrate to obtain the oxindole products $\mathbf{5 a} \sim \mathbf{5 0} . \mathbf{5 a} \sim \mathbf{5 0}$ were subjected to the acetylation reaction by the treatment with acetic anhydride in the presence of a catalytic amount of $N, N$-dimethylaminopyridine to afford the intermediates of 2-acetoxy-3-acetylindoles, which were used in next step without further purifucation. Subsequently, the hydrolysis reaction of the arising ester functions of the intermediates was carried out in tetrahydrofuran (THF) with the presence of $5 \%$ aq. $\mathrm{NaOH}$ at room temperature to give the targeted compounds $\mathbf{6 a} \sim \mathbf{6 0}$. Some compounds synthesized are novel. Their structures were confirmed by ${ }^{1} \mathrm{H}$ NMR, ${ }^{13} \mathrm{C}$ NMR, IR, MS and elemental analysis.

Keywords halo-functionalized isatin; reduction; alkylation; acetylation
\end{abstract}

已有研究和报道同时含有乙酰基和吲哚环结构的 化合物具有良好的药理活性和生物活性，是药物合成中 的一类重要的医药中间体. 从此类吲哚为起始原料已经 合成许多结构新颖的吲哚类杂环化合物, 并在医药行业 中得到广泛的应用, 显示了其广阔的发展前景, 人们已 经以此类化合物为医药中间体合成了具有抗人类免疫 缺陷病毒 $(\mathrm{HIV})^{[1 \sim 3]}$ 、抗病毒 ${ }^{[4]}$ 、抗增殖试剂 ${ }^{[5 \sim 8]}$ 、抗 癌 ${ }^{[9,10]}$ 、抗菌 ${ }^{[11]}$ 、抗细胞毒性 ${ }^{[12]}$ 、抗抑郁 ${ }^{[13]}$ 等各种药理 活性的含有吲哚环结构的药物或生物制剂, 因而其在药 物中间体的合成方面引起人们极大地研究兴趣. 因此我 们研究所一直不断的对吲哚及其衍生物的合成及性质 方面的研究进行探索. 为了继续深入研究结构新颖而有
趣的呐哚衍生物, 尽可能增强所合成的吲哚类化合物的 生物活性，我们对取代的乙酰基吲哚进行了一系列的研 究.

通过这些启示，我们设计合成的合成路线首先以对 卤代苯胺为原料合成卤素取代靛红然后与卤化烃合成 烃基取代的静红，随后烃基取代靛红经还原、酰化、水 解, 三步合成了预想的目标化合物 3-乙酰基-1-烃基-2羟基-5-卤吲哚, 这类化合物在药理研究或开发新的医 药产品可能具有重要的性质. 因此, 我们在此报道了吲 哚酮及吲哚衍生物的方法, 据我们所知, 至今为止还没 有相关文献报道此类吲哚衍生物的合成. 目标化合物 $\mathbf{6 a} \sim 60$ 的合成路线见 Scheme 1.

\footnotetext{
*E-mail: isfc@bhu.edu.cn

Received July 14, 2013; revised September 14, 2013; published online September 30.

Project supported by the Natural Science Foundation of Liaoning Province (No. 20120201).

辽宁省自然科学基金(No. 20120001)资助项目.
} 


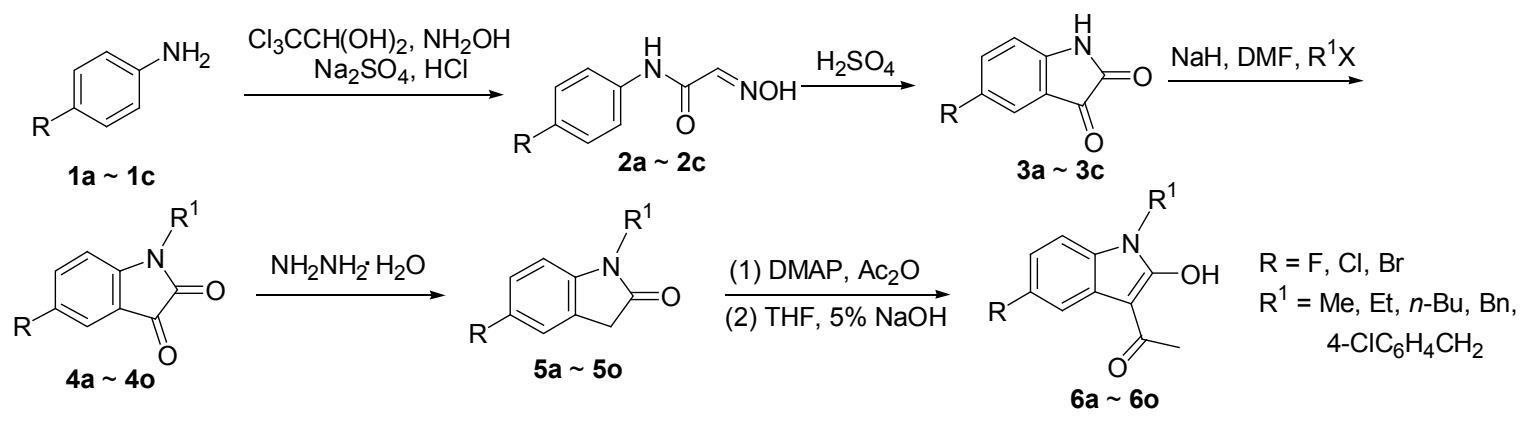

Scheme 1

\section{1 实验部分}

\section{1 仪器与试剂}

熔点用 WRS-1B 数字熔点仪测定(温度计未校正); IR 用 Nicolet AVAR 360 FT-IR 型傅里叶变换红外分光 光度计测定; 核磁共振氢谱采用 Agilent $1100-400 \mathrm{MHz}$ 型核磁共振仪测定, $\mathrm{CDCl}_{3}, \mathrm{DMSO}-d_{6}$ 为溶剂, TMS 为内 标; 质谱采用 Agilent1100 质谱仪测定; 元素分析由 Elementar Vario EL 测定. 所用的药品均为市售的分析 纯或化学纯或按照文献方法合成.

\section{2 卤代靛红的合成}

在装有电动摚拌、温度计和恒压滴管的三颈瓶中加 入 $40 \mathrm{~mL}$ 的水, 温热时加入无水硫酸钠 $(90.0 \mathrm{~g})$, 开动电 动搅拌使其均匀, 加入 $20 \mathrm{~mL}$ 的水合氯醛 $(0.06 \mathrm{~mol})$ 水溶 液, 然后缓慢滴加对卤代苯胺 $(\mathbf{1 a} \sim \mathbf{1 c})(0.04 \mathrm{~mol})$ 、浓盐 酸 $(10 \mathrm{~mL})$ 和水 $(70 \mathrm{~mL})$ 配制的溶液, 随着对卤代苯胺的 滴入将产生大量的絮状沉淀. 随后滴加盐酸着弪胺 $(0.13$ $\mathrm{mol})$ 的水溶液 $(40 \mathrm{~mL})$. 滴加完毕后升温至 $85{ }^{\circ} \mathrm{C}$. 用 $\mathrm{TLC}$ 监测反应进程, $1.5 \sim 2.5 \mathrm{~h}$ 后停止反应. 将反应体系 迅速冷却至室温, 抽滤、晾干, 得到粗产品为棕色固体. 用水重结晶, 均得到米白色针状晶体中间体.

向 $60{ }^{\circ} \mathrm{C}$ 温热的浓硫酸 $(15 \mathrm{~mL})$ 中少量多次分批加 入上述合成的中间体 $(0.02 \mathrm{~mol})$, 加料完毕后, 缓慢升温 至 $90{ }^{\circ} \mathrm{C}$, 保温 $30 \mathrm{~min}$, 反应过程中反应体系逐渐由棕 红色变为紫色. 冷却至室温后, 剧烈搅拌下将溶液倒入 $65 \mathrm{~mL}$ 水中, 冰浴冷却, 出现大量橘红色固体, 水解 $1 \mathrm{~h}$ 后, 抽滤, 水洗直至滤液呈中性, 晾干, 得粗产品, 经柱 层析分离得到纯品 $\mathbf{3 a} \sim \mathbf{3}$ [ 洗脱剂为 $V$ (乙酸乙酯)： $V$ (石 油醚 $)=1: 5]$.

5-氟静红(3a): 红色晶体, 产率 80.5\%. m.p. 231.4 $232.8{ }^{\circ} \mathrm{C}$ (文献值 ${ }^{[14]}$ : 产率 $74.2 \%$, m.p. $226.0 \sim 229.0{ }^{\circ} \mathrm{C}$ ); ${ }^{1} \mathrm{H}$ NMR (DMSO- $\left.d_{6}, 400 \mathrm{MHz}\right) \delta: 11.05(\mathrm{~s}, 1 \mathrm{H}, \mathrm{NH}$ ), $7.34 \sim 7.54$ (m, 2H, ArH), $6.92(\mathrm{~d}, J=8.6 \mathrm{~Hz}, 1 \mathrm{H}, \mathrm{ArH})$.

5-氯静红(3b): 黄色固体, 产率 78.6\%. m.p. 236.7 $239.5{ }^{\circ} \mathrm{C}$ (文献值 ${ }^{[15]}$ : 产率 $77.3 \%$, m.p. $237.8 \sim 239.1{ }^{\circ} \mathrm{C}$ ). 5-溴靛红(3c): 棕色固体, 产率 72.7\%. m.p. 260.3 $260.8{ }^{\circ} \mathrm{C}$ (文献值 ${ }^{[15]}$ : 产率 $70.0 \%$, m.p. $258.9 \sim 260.2{ }^{\circ} \mathrm{C}$ ).

\section{3 卤代靛红烃基化反应}

将甾素取代靛红(1 mmol)溶于 DMF (2 mL)中, 溶液 呈深红色. 在冰水浴条件下强力搅拌缓慢加入 $\mathrm{NaH}(1$ $\mathrm{mmol})$, 有大量气泡放出, 溶液变为紫色, 在冰水浴条 件下反应 $2 \mathrm{~h}$ 后，加入烷基化试剂(1.2 mmol)反应过程中 反应体系逐渐由紫色变为深红色, TLC 监测反应进程. 反应完后, 直接加入冰水中产生大量的红色沉淀, 调节 $\mathrm{pH}$ 至弱酸性, 抽滤, 晾干, 得粗产品, 用乙醇重结晶得 到纯品.

1-甲基-5-氟靛红(4a)：深红色晶体，产率 75.5\%. m.p. $157.5 \sim 158.1{ }^{\circ} \mathrm{C} ;{ }^{1} \mathrm{H}$ NMR $\left(\mathrm{CDCl}_{3}, 400 \mathrm{MHz}\right) \delta$ : $7.03 \sim 7.08(\mathrm{~m}, 2 \mathrm{H}, \mathrm{ArH}), 6.58 \sim 6.60(\mathrm{~m}, 1 \mathrm{H}, \mathrm{ArH}), 2.98$ (s, $\left.3 \mathrm{H}, \mathrm{CH}_{3}\right) ;{ }^{13} \mathrm{C}$ NMR $\left(\mathrm{CDCl}_{3}, 100 \mathrm{MHz}\right) \delta: 170.99$, $167.97,160.58,124.63,112.55,112.31,111.00,109.97$, 26.35; FT-IR (KBr) v: $1752,1705 \mathrm{~cm}^{-1}$; ESI-MS $m / z$ : $180.0[\mathrm{M}+\mathrm{H}]^{+}$. Anal. calcd for $\mathrm{C}_{9} \mathrm{H}_{6} \mathrm{FNO}_{2}: \mathrm{C} 60.34, \mathrm{H}$ 3.38, N 7.82; found C 60.45, H 3.44, N 7.72.

1-乙基-5-氟静红(4b): 红色晶体, 产率 62.2\%. m.p. $128.0 \sim 129.3{ }^{\circ} \mathrm{C} ;{ }^{1} \mathrm{H}$ NMR $\left(\mathrm{CDCl}_{3}, 400 \mathrm{MHz}\right) \delta: 7.22 \sim$ 7.27 (m, $2 \mathrm{H}, \mathrm{ArH}), 6.79 \sim 6.83(\mathrm{~m}, 1 \mathrm{H}, \mathrm{ArH}), 3.66 \sim 3.76$ (m, $\left.2 \mathrm{H}, \mathrm{CH}_{2}\right), 1.24(\mathrm{t}, J=7.2 \mathrm{~Hz}, 3 \mathrm{H}) ;{ }^{13} \mathrm{C} \mathrm{NMR}\left(\mathrm{CDCl}_{3}\right.$, $100 \mathrm{MHz}) \delta$ : $160.42,157.98,146.65,124.70,124.46$, 112.70, 112.45, 111.13, 111.06, 35.05, 12.37; FT-IR (KBr) $v: 1754,1707 \mathrm{~cm}^{-1}$; ESI-MS $m / z: 194.0[\mathrm{M}+\mathrm{H}]^{+}$. Anal. calcd for $\mathrm{C}_{10} \mathrm{H}_{8} \mathrm{FNO}_{2}$ : C 62.18, H 4.17, N 7.25; found $\mathrm{C}$ 62.24, H 4.25, N 7.12.

1-正丁基-5-氟靛红(4c)：红色晶体，产率 55.5\%. m.p. $75.5 \sim 77.6{ }^{\circ} \mathrm{C} ;{ }^{1} \mathrm{H} \mathrm{NMR}\left(\mathrm{CDCl}_{3}, 400 \mathrm{MHz}\right) \delta$ : $7.10 \sim$ $7.35(\mathrm{~m}, 2 \mathrm{H}, \mathrm{ArH}), 6.70 \sim 6.88(\mathrm{~m}, 1 \mathrm{H}, \mathrm{ArH}), 3.65(\mathrm{t}, J=$ $\left.7.3 \mathrm{~Hz}, 2 \mathrm{H}, \mathrm{CH}_{2}\right), 1.56 \sim 1.66\left(\mathrm{~m}, 2 \mathrm{H}, \mathrm{CH}_{2}\right), 1.34(\mathrm{dt}, J=$ $\left.7.5 \mathrm{~Hz}, 2 \mathrm{H}, \mathrm{CH}_{2}\right), 0.90\left(\mathrm{t}, J=6.7 \mathrm{~Hz}, 3 \mathrm{H}, \mathrm{CH}_{3}\right) ;{ }^{13} \mathrm{C} \mathrm{NMR}$ $\left(\mathrm{CDCl}_{3}, 100 \mathrm{MHz}\right) \delta: 160.40,157.95,124.55,118.10$, 112.61, 112.37, 111.25, 109.99, 40.10, 29.15, 20.09, 13.65; 
FT-IR (KBr) v: 1752, $1702 \mathrm{~cm}^{-1}$; ESI-MS m/z: $222.0[\mathrm{M}+$ $\mathrm{H}]^{+}$. Anal. calcd for $\mathrm{C}_{12} \mathrm{H}_{12} \mathrm{FNO}_{2}$ : C 65.15, H 5.47, N 6.33; found C 65.23, H 5.56, N 6.18 .

1-苠基-5-氟靛红(4d): 红色晶体, 产率 82.4\%. m.p. $133.1 \sim 134.2{ }^{\circ} \mathrm{C}$ (文献值 ${ }^{[16]}$ : 产率 $80.0 \%$, m.p. 130.0 $\left.133.0{ }^{\circ} \mathrm{C}\right) ;{ }^{1} \mathrm{H}$ NMR $\left(\mathrm{CDCl}_{3}, 400 \mathrm{MHz}\right) \delta: 7.29 \sim 7.36(\mathrm{~m}$, $6 \mathrm{H}, \mathrm{ArH}), 7.15 \sim 7.20(\mathrm{~m}, 1 \mathrm{H}, \mathrm{ArH}), 6.70 \sim 6.73(\mathrm{~m}, 1 \mathrm{H}$, $\mathrm{ArH}), 4.91\left(\mathrm{~s}, 2 \mathrm{H}, \mathrm{CH}_{2}\right) ;{ }^{13} \mathrm{C} \mathrm{NMR}\left(\mathrm{CDCl}_{3}, 100 \mathrm{MHz}\right) \delta$ : $166.14,163.69$, 152.32, 139.70, 134.73, 133.90, 132.97, 130.36, 130.12, 118.17, 117.92, 117.84, 117.77, 49.76; FT-IR (KBr) $v: 1755,1708 \mathrm{~cm}^{-1}$; ESI-MS m/z: $256.0[\mathrm{M}+$ $\mathrm{H}]^{+}$. Anal. calcd for $\mathrm{C}_{15} \mathrm{H}_{10} \mathrm{FNO}_{2}$ : C 70.58, H 3.95, N 5.49; found $\mathrm{C} 70.64, \mathrm{H} 4.05, \mathrm{~N} 5.63$.

1-对氯苄基-5-氟靛红(4e)：棕红色晶体，产率 90.1\%. m.p. $155.2 \sim 156.1{ }^{\circ} \mathrm{C} ;{ }^{1} \mathrm{H}$ NMR $\left(\mathrm{CDCl}_{3}, 400\right.$ $\mathrm{MHz}) \delta: 7.32(\mathrm{~d}, J=8.5 \mathrm{~Hz}, 2 \mathrm{H}, \mathrm{ArH}), 7.14 \sim 7.29(\mathrm{~m}, 4 \mathrm{H}$, ArH), 6.68 (d, J=8.6 Hz, 1H, ArH), $4.88\left(\mathrm{~s}, 2 \mathrm{H}, \mathrm{CH}_{2}\right) ;{ }^{13} \mathrm{C}$ NMR $\left(\mathrm{CDCl}_{3}, 100 \mathrm{MHz}\right) \delta: 205.69,139.87,138.23$, $134.95,134.36,130.41,130.23,118.36,118.13,117.62$, 117.55, 98.20, 49.13; FT-IR (KBr) v: 1758, $1710 \mathrm{~cm}^{-1}$; ESI-MS $m / z: 290.0[\mathrm{M}+\mathrm{H}]^{+}$. Anal. calcd for $\mathrm{C}_{15} \mathrm{H}_{9} \mathrm{ClFNO}_{2}$ : C 62.19, H 3.13, N 4.84; found C 62.24, H $3.21, \mathrm{~N} 4.64$

1-甲基-5-氯靛红(4f)：红色晶体，产率 90.5\%. m.p. $135.9 \sim 136.5{ }^{\circ} \mathrm{C} ;{ }^{1} \mathrm{H}$ NMR $\left(\mathrm{CDCl}_{3}, 400 \mathrm{MHz}\right) \delta: 7.48 \sim$ 7.52 (m, 2H, ArH), 6.82 (d, J=8.1 Hz, 1H, ArH), 3.19 (s, $\left.3 \mathrm{H}, \mathrm{CH}_{3}\right) ;{ }^{13} \mathrm{C} \mathrm{NMR}\left(\mathrm{CDCl}_{3}, 100 \mathrm{MHz}\right) \delta: 180.52,155.81$, 147.83, 135.95, 127.78, 123.29, 116.31, 109.46, 24.55; FT-IR (KBr) v: 1751, $1709 \mathrm{~cm}^{-1}$; ESI-MS m/z: $196.0[\mathrm{M}+$ $\mathrm{H}]^{+}$. Anal. calcd for $\mathrm{C}_{9} \mathrm{H}_{6} \mathrm{ClNO}_{2}$ : C 55.26, H 3.09, N 7.16; found $\mathrm{C}$ 55.32, H 3.12, N 7.02 .

1-乙基-5-氯靛红(4g): 红色晶体, 产率 95.4\%. m.p. $135.9 \sim 136.5{ }^{\circ} \mathrm{C} ;{ }^{1} \mathrm{H}$ NMR $\left(\mathrm{CDCl}_{3}, 400 \mathrm{MHz}\right) \delta: 7.50(\mathrm{~s}$, $2 \mathrm{H}, \mathrm{ArH}), 6.82(\mathrm{~d}, J=8.8 \mathrm{~Hz}, 1 \mathrm{H}, \mathrm{ArH}), 3.72$ (dd, $J=7.1$, $\left.14.4 \mathrm{~Hz}, 2 \mathrm{H}, \mathrm{CH}_{2}\right), 1.24\left(\mathrm{t}, J=7.2 \mathrm{~Hz}, 3 \mathrm{H}, \mathrm{CH}_{3}\right) ;{ }^{13} \mathrm{C} \mathrm{NMR}$ $\left(\mathrm{CDCl}_{3}, 100 \mathrm{MHz}\right) \delta: 182.65,157.27,148.87,137.66$, 129.43, 125.40, 118.39, 111.27, 35.12, 12.44; FT-IR (KBr) $v: 1753,1712 \mathrm{~cm}^{-1}$; ESI-MS m/z: $209.0[\mathrm{M}+\mathrm{H}]^{+}$. Anal. calcd for $\mathrm{C}_{10} \mathrm{H}_{8} \mathrm{ClNO}_{2}$ : C 57.30, H $3.85 \mathrm{~N} \mathrm{6.68;} \mathrm{found} \mathrm{C}$ 57.41, H 3.94, N 6.54.

1-正丁基-5-氯靛红(4h)：红色晶体，产率 75.5\%. m.p. $78.4 \sim 80.0{ }^{\circ} \mathrm{C} ;{ }^{1} \mathrm{H}$ NMR $\left(\mathrm{CDCl}_{3}, 400 \mathrm{MHz}\right) \delta$ : 7.98 (d, $J=7.1 \mathrm{~Hz}, 2 \mathrm{H}, \mathrm{ArH}), 7.30$ (d, $J=8.7 \mathrm{~Hz}, 1 \mathrm{H}, \mathrm{ArH})$, $4.15(\mathrm{t}, J=7.2 \mathrm{~Hz}, 2 \mathrm{H}, \mathrm{ArH}), 2.05 \sim 2.15\left(\mathrm{~m}, 2 \mathrm{H}, \mathrm{CH}_{2}\right)$, 1.84 (dt, $\left.J=7.4,14.9 \mathrm{~Hz}, 2 \mathrm{H}, \mathrm{CH}_{2}\right), 1.40(\mathrm{t}, J=7.3 \mathrm{~Hz}$, $\left.3 \mathrm{H}, \mathrm{CH}_{3}\right) ;{ }^{13} \mathrm{C} \mathrm{NMR}\left(\mathrm{CDCl}_{3}, 100 \mathrm{MHz}\right) \delta: 180.81,155.73$, $147.45,135.82,127.55,123.50,123.44,109.64,38.32$, 27.34, 18.26, 11.82; FT-IR (KBr) v: 1756, $1707 \mathrm{~cm}^{-1}$; ESI-MS $m / z: 238.0[\mathrm{M}+\mathrm{H}]^{+}$. Anal. calcd for $\mathrm{C}_{12} \mathrm{H}_{12} \mathrm{Cl}-$ $\mathrm{NO}_{2}$ : C 60.64, H 5.09, N 5.89; found $\mathrm{C} 60.73, \mathrm{H} \mathrm{5.12,} \mathrm{N}$ 5.75 .

1-苠基-5-氯靛红(4i)：红色晶体，产率 94.1\%. m.p. $141.9 \sim 142.5{ }^{\circ} \mathrm{C} ;{ }^{1} \mathrm{H}$ NMR $\left(\mathrm{CDCl}_{3}, 400 \mathrm{MHz}\right) \delta: 7.63(\mathrm{~s}$, $1 \mathrm{H}, \mathrm{ArH}), 7.50$ (s, 1H, ArH), 7.24 (s, 5H, ArH), 6.60 (s, $1 \mathrm{H}, \mathrm{ArH}), 4.85$ (s, 2H, $\left.\mathrm{CH}_{2}\right) ;{ }^{13} \mathrm{C} \mathrm{NMR}\left(\mathrm{CDCl}_{3}, 100 \mathrm{MHz}\right)$ $\delta: 182.24,157.68,150.69,148.89,137.66,133.99,129.71$, $129.13,128.32,127.37,125.28,118.38,112.31,109.98$, 44.15; FT-IR (KBr) v: 1760, $1701 \mathrm{~cm}^{-1}$; ESI-MS $\mathrm{m} / \mathrm{z}$ : $272.0[\mathrm{M}+\mathrm{H}]^{+}$. Anal. calcd for $\mathrm{C}_{15} \mathrm{H}_{10} \mathrm{ClNO}_{2}: \mathrm{C} 66.31, \mathrm{H}$ 3.71, N 5.16; found C 66.38, H 3.82, N 5.01.

1-对氯苄基-5-氯靛红(4j)：红色晶体，产率 $97.5 \%$. m.p. $188.8 \sim 189.6{ }^{\circ} \mathrm{C} ;{ }^{1} \mathrm{H}$ NMR $\left(\mathrm{CDCl}_{3}, 400 \mathrm{MHz}\right) \delta$ : 7.51 (s, 1H, ArH), 7.39 (d, $J=8.2 \mathrm{~Hz}, 1 \mathrm{H}, \operatorname{ArH}), 7.26$ (d, $J=7.8 \mathrm{~Hz}, 2 \mathrm{H}, \mathrm{ArH}), 7.19$ (d, $J=7.6 \mathrm{~Hz}, 2 \mathrm{H}, \mathrm{ArH}), 6.63$ (d, $J=8.3 \mathrm{~Hz}, 1 \mathrm{H}, \mathrm{ArH}), 4.83\left(\mathrm{~s}, 2 \mathrm{H}, \mathrm{CH}_{2}\right) ;{ }^{13} \mathrm{C} \mathrm{NMR}$ $\left(\mathrm{CDCl}_{3}, 100 \mathrm{MHz}\right) \delta: 180.15,155.82,146.73,135.90$, $132.46,130.71,128.10,127.52$, 126.94, 123.65, 116.63, 110.27, 41.70; FT-IR (KBr) v: 1756, $1703 \mathrm{~cm}^{-1}$; ESI-MS $m / z: 307.0[\mathrm{M}+\mathrm{H}]^{+}$. Anal. calcd for $\mathrm{C}_{15} \mathrm{H}_{9} \mathrm{Cl}_{2} \mathrm{NO}_{2}: \mathrm{C}$ 58.85, H 2.96, N 4.58; found C 58.90, H 2.97, N 4.41.

1-甲基-5-溴靛红(4k)：红色晶体，产率 90.1\%. m.p. $172.7 \sim 174.2{ }^{\circ} \mathrm{C} ;{ }^{1} \mathrm{H}$ NMR $\left(\mathrm{CDCl}_{3}, 400 \mathrm{MHz}\right) \delta: 7.63 \sim$ $7.66(\mathrm{~m}, 2 \mathrm{H}, \mathrm{ArH}), 6.75$ (d, J=8.2 Hz, 1H, ArH), 3.19 (s, $\left.3 \mathrm{H}, \mathrm{CH}_{3}\right) ;{ }^{13} \mathrm{C} \mathrm{NMR}\left(\mathrm{CDCl}_{3}, 100 \mathrm{MHz}\right) \delta: 180.66,155.64$, $148.27,138.78,126.17,116.68,114.82,109.83,24.57$ FT-IR (KBr) $v: 1762,1714 \mathrm{~cm}^{-1}$; ESI-MS m/z: $241.0[\mathrm{M}+$ $\mathrm{H}]^{+}$. Anal. calcd for $\mathrm{C}_{9} \mathrm{H}_{6} \mathrm{BrNO}_{2}$ : C 45.03, $\mathrm{H}$ 2.52, N 5.83; found C 45.13, H 2.53, N 5.64.

1-乙基-5-溴靛红(41): 红色晶体，产率 $83.6 \%$. m.p. $147.6 \sim 149.2{ }^{\circ} \mathrm{C}$ (文献值 ${ }^{[17]}$ 产率 $50.0 \%$ ); ${ }^{1} \mathrm{H}$ NMR $\left(\mathrm{CDCl}_{3}, 400 \mathrm{MHz}\right) \delta: 7.64(\mathrm{~d}, J=9.3 \mathrm{~Hz}, 2 \mathrm{H}, \mathrm{ArH}), 6.77$ (d, $J=8.1 \mathrm{~Hz}, 1 \mathrm{H}, \mathrm{ArH}), 3.71\left(\mathrm{q}, J=6.9 \mathrm{~Hz}, 2 \mathrm{H}, \mathrm{CH}_{2}\right)$, $1.24\left(\mathrm{t}, J=7.0 \mathrm{~Hz}, 3 \mathrm{H}, \mathrm{CH}_{3}\right) ;{ }^{13} \mathrm{C} \mathrm{NMR}\left(\mathrm{CDCl}_{3}, 100 \mathrm{MHz}\right)$ $\delta: 180.69,155.27,147.49,138.75,126.34,116.89,114.58$, 109.92, 33.29, 10.60; FT-IR (KBr) v: 1763, $1712 \mathrm{~cm}^{-1}$; ESI-MS $m / z: 255.0[\mathrm{M}+\mathrm{H}]^{+}$. Anal. calcd for $\mathrm{C}_{10} \mathrm{H}_{8} \mathrm{BrNO}_{2}$ : C 47.27, H 3.17, N 5.51; found C 47.33, H 3.21, N 5.43.

1-正丁基-5-溴靛红(4m)：红色晶体，产率 $86.7 \%$. m.p. 95.9 96.4 ${ }^{\circ} \mathrm{C} ;{ }^{1} \mathrm{H}$ NMR $\left(\mathrm{CDCl}_{3}, 400 \mathrm{MHz}\right) \delta: 7.63$ (s, 2H, ArH), 6.77 (s, 1H, ArH), 3.65 (s, 2H, $\left.\mathrm{CH}_{2}\right), 1.60$ (s, 
2H, $\mathrm{CH}_{2}$ ), 1.34 (s, 2H, $\mathrm{CH}_{2}$ ), 1.18 (s, 2H, $\left.\mathrm{CH}_{2}\right), 0.90$ (s, $\left.3 \mathrm{H}, \mathrm{CH}_{3}\right) ;{ }^{13} \mathrm{C} \mathrm{NMR}\left(\mathrm{CDCl}_{3}, 100 \mathrm{MHz}\right) \delta: 180.64$, 155.54, 147.89, 138.66, 126.30, 116.86, 114.55, 110.05, 38.31, 27.50, 18.26, 11.82; FT-IR (KBr) v: 1756, 1716 $\mathrm{cm}^{-1}$; ESI-MS $m / z: 283.0[\mathrm{M}+\mathrm{H}]^{+}$. Anal. calcd for $\mathrm{C}_{12} \mathrm{H}_{12} \mathrm{BrNO}_{2}$ : C 51.09, H 4.29, N 4.96; found C 51.12, H $4.35, \mathrm{~N} 4.78$.

1-芐基-5-溴静红(4n): 红色晶体, 产率 90.2\%. m.p. 153.4 155.3 ${ }^{\circ} \mathrm{C} ;{ }^{1} \mathrm{H}$ NMR $\left(\mathrm{CDCl}_{3}, 400 \mathrm{MHz}\right) \delta: 7.63$ (s, 1H, ArH), 7.50 (s, 1H, ArH), 7.24 (s, 5H, ArH), 6.60 (s, $1 \mathrm{H}, \mathrm{ArH}), 4.85$ (s, 2H, $\left.\mathrm{CH}_{2}\right) ;{ }^{13} \mathrm{C} \mathrm{NMR}\left(\mathrm{CDCl}_{3}, 100 \mathrm{MHz}\right)$ $\delta: 182.06,157.49,149.33,140.56,133.84,129.13,128.92$, $128.33,128.14,127.36,127.15,118.78,116.75,112.70$, 44.14; FT-IR (KBr) $v$ : $1755,1710 \mathrm{~cm}^{-1}$; ESI-MS $\mathrm{m} / z$ : $317.0[\mathrm{M}+\mathrm{H}]^{+}$. Anal. calcd for $\mathrm{C}_{15} \mathrm{H}_{10} \mathrm{BrNO}_{2}: \mathrm{C} 56.99, \mathrm{H}$ 3.19, N 4.43; found C 57.04, H 3.23, N 4.28.

1-对氯芐基-5-溴静红(40)：红色晶体，产率 70.3\%. m.p. $207.7 \sim 208.2{ }^{\circ} \mathrm{C} ;{ }^{1} \mathrm{H}$ NMR $\left(400 \mathrm{MHz}, \mathrm{CDCl}_{3}\right) \delta$ : 7.66 (s, $1 \mathrm{H}, \operatorname{ArH}), 7.54(\mathrm{~d}, J=8.3 \mathrm{~Hz}, 1 \mathrm{H}, \mathrm{ArH}), 7.26$ (d, $J=8.2 \mathrm{~Hz}, 2 \mathrm{H}, \mathrm{ArH}), 7.18(\mathrm{~d}, J=8.0 \mathrm{~Hz}, 2 \mathrm{H}, \mathrm{ArH}), 6.58$ (d, $J=8.3 \mathrm{~Hz}, 1 \mathrm{H}, \mathrm{ArH}), 4.82\left(\mathrm{~s}, 2 \mathrm{H}, \mathrm{CH}_{2}\right) ;{ }^{13} \mathrm{C} \mathrm{NMR}(100$ $\left.\mathrm{MHz}, \mathrm{CDCl}_{3}\right) \delta: 179.97,147.17,138.76,132.49,130.66$, $127.53,126.93,116.97,115.14,110.65,41.69$; FT-IR (KBr) $v$ : $1757,1712 \mathrm{~cm}^{-1}$; ESI-MS $m / z: 351.0[\mathrm{M}+\mathrm{H}]^{+}$. Anal. calcd for $\mathrm{C}_{15} \mathrm{H}_{9} \mathrm{BrClNO}_{2}$ : C 51.39, H 2.59, $\mathrm{N} 4.00$; found C 51.43, H 2.65, N 3.87 .

\section{$1.4 N$-烃基卤代靛红还原反应}

1-烃基-5-卤代靛红(1 mmol)溶于 $85 \%$ 水合肼(1.5 $\mathrm{mL})$ 中, 在 $150{ }^{\circ} \mathrm{C}$ 反应条件下回流反应, TLC 监测反应 进程, 反应结束后直接加入冰水, 调节 $\mathrm{pH}$ 至弱酸性, 析 出的沉淀经抽滤、干燥得粗产品, 用乙醇重结晶得到纯 品.

1-甲基-5-氟吲哚酮 $(5 a)$ : 淡黄色晶体, 产率 $82.5 \%$. m.p. 129.6 130 ${ }^{\circ} \mathrm{C} ;{ }^{1} \mathrm{H}$ NMR $\left(400 \mathrm{MHz}, \mathrm{CDCl}_{3}\right) \delta$ : 7.03 (d, $J=3.7 \mathrm{~Hz}, 2 \mathrm{H}, \mathrm{ArH}), 6.62 \sim 6.67(\mathrm{~m}, 1 \mathrm{H}, \mathrm{ArH}), 3.21$ (s, $\left.2 \mathrm{H}, \mathrm{CH}_{2}\right) 2.98\left(\mathrm{~s}, 3 \mathrm{H}, \mathrm{CH}_{3}\right) ;{ }^{13} \mathrm{C} \mathrm{NMR}\left(\mathrm{CDCl}_{3}, 100\right.$ MHz) $\delta: 170.72,153.08,129.77,116.14,114.60,110.50$, 108.96, 38.10, 28.42; FT-IR (KBr) $v: 1705 \mathrm{~cm}^{-1}$; ESI-MS $m / z: 166.0[\mathrm{M}+\mathrm{H}]^{+}$. Anal. calcd for $\mathrm{C}_{9} \mathrm{H}_{8} \mathrm{FNO}: \mathrm{C} 65.45, \mathrm{H}$ 4.88, N 8.48; found C 65.45, H 4.88, N 8.48.

1-乙基-5-氟吲哚酮 $(\mathbf{5 b})$ : 米白色晶体, 产率 $73.2 \%$. m.p. $113.0 \sim 113.4{ }^{\circ} \mathrm{C} ;{ }^{1} \mathrm{H}$ NMR $\left(\mathrm{CDCl}_{3}, 400 \mathrm{MHz}\right) \delta$ : $6.88 \sim 6.94(\mathrm{~m}, 2 \mathrm{H}, \operatorname{ArH}), 6.66 \sim 6.69(\mathrm{~m}, 1 \mathrm{H}, \operatorname{ArH})$, $3.62 \sim 3.74\left(\mathrm{~m}, 2 \mathrm{H}, \mathrm{CH}_{2}\right), 3.44\left(\mathrm{~s}, 2 \mathrm{H}, \mathrm{CH}_{2}\right), 1.18$ (t, $J=$ $\left.7.2 \mathrm{~Hz}, 3 \mathrm{H}, \mathrm{CH}_{3}\right) ;{ }^{13} \mathrm{C} \mathrm{NMR}\left(\mathrm{CDCl}_{3}, 100 \mathrm{MHz}\right) \delta: 173.15$,
159.02, 113.02, 112.79, 111.71, 111.46, 107.40, 35.01, 33.72, 11.53; FT-IR (KBr) $v: 1703 \mathrm{~cm}^{-1}$; ESI-MS m/z: $180.0[\mathrm{M}+\mathrm{H}]^{+}$. Anal. calcd for $\mathrm{C}_{10} \mathrm{H}_{10} \mathrm{FNO}$ : C 67.03, $\mathrm{H}$ 5.62, N 7.82; found C 67.13, H 5.73, N 7.67.

1-正丁基-5-氟吗哚酩(5c): 白色晶体，产率 $80.2 \%$. m.p. $37.9 \sim 38.2{ }^{\circ} \mathrm{C} ;{ }^{1} \mathrm{H}$ NMR $\left(\mathrm{CDCl}_{3}, 400 \mathrm{MHz}\right) \delta$ : 6.93 (dd, $J=18.2,8.6 \mathrm{~Hz}, 2 \mathrm{H}, \mathrm{ArH}), 6.70$ (d, $J=3.6 \mathrm{~Hz}, 1 \mathrm{H}$, $\mathrm{ArH}$ ), 3.64 (t, $\left.J=6.4 \mathrm{~Hz}, 2 \mathrm{H}, \mathrm{CH}_{2}\right), 3.46\left(\mathrm{~s}, 2 \mathrm{H}, \mathrm{CH}_{2}\right.$ ), $1.51 \sim 1.70\left(\mathrm{~m}, 2 \mathrm{H}, \mathrm{CH}_{2}\right), 1.25 \sim 1.43\left(\mathrm{~m}, 2 \mathrm{H}, \mathrm{CH}_{2}\right), 0.91$ (t, $\left.J=7.3 \mathrm{~Hz}, 3 \mathrm{H}, \mathrm{CH}_{2}\right) ;{ }^{13} \mathrm{C} \mathrm{NMR}\left(\mathrm{CDCl}_{3}, 100 \mathrm{MHz}\right) \delta$ : 174.42, 160.00, 157.62, 140.56, 126.18, 113.87, 112.51, 108.56, 39.86, 35.95, 29.37, 20.15, 13.72; FT-IR (KBr) v: $1708 \mathrm{~cm}^{-1}$; ESI-MS $m / z: 208.0[\mathrm{M}+\mathrm{H}]^{+}$. Anal. calcd for $\mathrm{C}_{12} \mathrm{H}_{14} \mathrm{FNO}$ : C 69.55, H 6.81, N 6.76; found C 69.67, H 6.94, N 6.58 .

1-芐基-5-氟吲哚酮 (5d): 淡黄色粉末, 产率 81.2\%. m.p. $76.5 \sim 77.1{ }^{\circ} \mathrm{C} ;{ }^{1} \mathrm{H}$ NMR $\left(\mathrm{CDCl}_{3}, 400 \mathrm{MHz}\right) \delta$ : $7.18 \sim 7.26$ (m, 5H, ArH), $6.92(\mathrm{~d}, J=7.7 \mathrm{~Hz}, 1 \mathrm{H}, \operatorname{ArH})$, $6.78(\mathrm{t}, J=8.8 \mathrm{~Hz}, 1 \mathrm{H}, \mathrm{ArH}), 6.53(\mathrm{dd}, J=8.1,3.8 \mathrm{~Hz}, 1 \mathrm{H}$, $\mathrm{ArH}), 4.82\left(\mathrm{~s}, 2 \mathrm{H}, \mathrm{CH}_{2}\right), 3.54\left(\mathrm{~s}, 2 \mathrm{H}, \mathrm{CH}_{2}\right) ;{ }^{13} \mathrm{C} \mathrm{NMR}$ $\left(\mathrm{CDCl}_{3}, 100 \mathrm{MHz}\right) \delta: 174.65,160.20,140.17,135.53$, $128.80,127.71,127.28,114.12,113.89,112.65,112.41$, 109.47, 109.39, 43.85, 36.00; FT-IR (KBr) $v: 1706 \mathrm{~cm}^{-1}$; ESI-MS $m / z: 242.0[\mathrm{M}+\mathrm{H}]^{+}$. Anal. calcd for $\mathrm{C}_{12} \mathrm{H}_{14} \mathrm{FNO}$ : C 69.55, H 6.81, N 6.76; found C 69.67, H 6.92, N 6.54.

1-对氯芐基-5-氟吲哚酮 (5e): 淡黄色固体, 产率 92.6\%. m.p. 133.5 134.2 ${ }^{\circ} \mathrm{C} ;{ }^{1} \mathrm{H}$ NMR $\left(\mathrm{CDCl}_{3}, 400\right.$ MHz) $\delta: 7.30 \sim 7.07(\mathrm{~m}, 6 \mathrm{H}, \mathrm{ArH}), 6.94(\mathrm{~d}, J=4.1 \mathrm{~Hz}, 1 \mathrm{H}$, ArH), 4.88 (t, $J=9.9 \mathrm{~Hz}, 2 \mathrm{H}, \mathrm{CH}_{2}$ ), 4.79 (s, $2 \mathrm{H}, \mathrm{CH}_{2}$ ), 3.55 (s, $\left.2 \mathrm{H}, \mathrm{CH}_{2}\right) ;{ }^{13} \mathrm{C}$ NMR $\left(\mathrm{CDCl}_{3}, 100 \mathrm{MHz}\right) \delta: 169.86$, $155.53,135.11,129.31,128.83,124.24,123.93,121.22$, $109.45,109.21,108.06,107.81,104.55,38.48,31.20$; FT-IR (KBr) $v: 1702 \mathrm{~cm}^{-1}$; ESI-MS $m / z: 276.0[\mathrm{M}+\mathrm{H}]^{+}$. Anal. calcd for $\mathrm{C}_{15} \mathrm{H}_{11} \mathrm{ClFNO}$ : C 65.35, H 4.02, N 5.08; found C $65.45, \mathrm{H} 4.21, \mathrm{~N} 4.89$.

1-甲基-5-氯吲哚酮 $(\mathbf{5 f})$ : 黄色固体, 产率 $84.0 \%$. m.p. $120.6 \sim 121.5{ }^{\circ} \mathrm{C} ;{ }^{1} \mathrm{H}$ NMR $\left(\mathrm{CDCl}_{3}, 400 \mathrm{MHz}\right) \delta$ : $7.20(\mathrm{~d}, J=8.6 \mathrm{~Hz}, 2 \mathrm{H}, \mathrm{ArH}), 6.70(\mathrm{~d}, J=8.2 \mathrm{~Hz}, 1 \mathrm{H}$, $\mathrm{ArH}), 3.48\left(\mathrm{~s}, 2 \mathrm{H}, \mathrm{CH}_{2}\right), 3.17$ (s, $\left.3 \mathrm{H}, \mathrm{CH}_{3}\right) ;{ }^{13} \mathrm{C} \mathrm{NMR}$ $\left(\mathrm{CDCl}_{3}, 100 \mathrm{MHz}\right) \delta: 174.40,143.72,127.78,127.62$, 126.01, 124.74, 108.85, 35.61, 26.27; FT-IR (KBr) v: 1710 $\mathrm{cm}^{-1}$; ESI-MS $m / z: 182.0[\mathrm{M}+\mathrm{H}]^{+}$. Anal. calcd for $\mathrm{C}_{9} \mathrm{H}_{8} \mathrm{ClNO}$ : C 59.52, H 4.44, N 7.71; found $\mathrm{C} 59.63, \mathrm{H}$ 4.52, N 7.41.

1-乙基-5-氯吲哚酮 $(5 \mathrm{~g})$ ：米白色晶体，产率 $86.1 \%$. 
m.p. $120.6 \sim 121.5{ }^{\circ} \mathrm{C} ;{ }^{1} \mathrm{H}$ NMR $\left(\mathrm{CDCl}_{3}, 400 \mathrm{MHz}\right) \delta$ : 7.17 (d, $J=10.4 \mathrm{~Hz}, 2 \mathrm{H}, \operatorname{ArH}), 6.69$ (d, $J=10.1 \mathrm{~Hz}, 1 \mathrm{H}$, ArH), 3.68 (q, $\left.J=6.9 \mathrm{~Hz}, 2 \mathrm{H}, \mathrm{CH}_{2}\right), 3.45$ (d, $J=13.4 \mathrm{~Hz}$, $\left.2 \mathrm{H}, \mathrm{CH}_{2}\right), 1.18\left(\mathrm{t}, J=7.0 \mathrm{~Hz}, 3 \mathrm{H}, \mathrm{CH}_{3}\right) ;{ }^{13} \mathrm{C} \mathrm{NMR}\left(\mathrm{CDCl}_{3}\right.$, $100 \mathrm{MHz}) \delta: 174.03,142.81,127.70,127.40,126.31$, 124.92, 108.94, 35.70, 34.76, 12.56; FT-IR (KBr) v: 1705 $\mathrm{cm}^{-1}$; ESI-MS $m / z: 196.0[\mathrm{M}+\mathrm{H}]^{+}$. Anal. calcd for $\mathrm{C}_{10} \mathrm{H}_{10} \mathrm{ClNO}$ : C 61.39, H 5.15, N 7.16; found C 61.51, H 5.23, N 7.03 .

1-正丁基-5-氯吲哚酮 (5h): 淡黄色晶体, 产率 76.2\%. m.p. 52.0 52.3 ${ }^{\circ} \mathrm{C} ;{ }^{1} \mathrm{H}$ NMR $\left(\mathrm{CDCl}_{3}, 400 \mathrm{MHz}\right)$ $\delta: 7.20$ (s, 2H, ArH), 6.75 (s, 1H, ArH), 3.67 (d, $J=6.9 \mathrm{~Hz}$, $2 \mathrm{H}, \mathrm{CH}_{2}$ ), 3.49 (s, 2H, $\mathrm{CH}_{2}$ ), 1.62 (dd, $J=6.9,13.8 \mathrm{~Hz}$, $\left.2 \mathrm{H}, \mathrm{CH}_{2}\right), 1.35\left(\mathrm{dd}, J=7.1,14.3 \mathrm{~Hz}, 2 \mathrm{H}, \mathrm{CH}_{2}\right), 0.97 \sim 0.88$ $\left(\mathrm{m}, 3 \mathrm{H}, \mathrm{CH}_{3}\right) ;{ }^{13} \mathrm{C} \mathrm{NMR}\left(\mathrm{CDCl}_{3}, 100 \mathrm{MHz}\right) \delta: 174.26$, $143.21,127.68,127.38,126.25$, 124.87, 108.95, 39.91, 35.67, 29.39, 20.17, 13.74; FT-IR (KBr) $v: 1703 \mathrm{~cm}^{-1}$; ESI-MS $m / z: 224.0[\mathrm{M}+\mathrm{H}]^{+}$. Anal. calcd for $\mathrm{C}_{12} \mathrm{H}_{14} \mathrm{CINO}$ : C 64.43, H 6.31, N 6.26; found C 64.57, H 6.41, N 6.03.

1-苠基-5-氯吲哚酮(5i): 淡黄色固体, 产率 $85.1 \%$. m.p. 102.6 $104.3{ }^{\circ} \mathrm{C}$ (文献值 ${ }^{[18]}$ : 产率 76.0\%); ${ }^{1} \mathrm{H} \mathrm{NMR}$ $\left(\mathrm{CDCl}_{3}, 400 \mathrm{MHz}\right) \delta: 7.48 \sim 7.11(\mathrm{~m}, 6 \mathrm{H}, \mathrm{ArH}), 7.06(\mathrm{~d}$, $J=8.3 \mathrm{~Hz}, 1 \mathrm{H}, \mathrm{ArH}), 6.54$ (d, $J=8.4 \mathrm{~Hz}, 1 \mathrm{H}, \mathrm{ArH}), 4.83$ $\left(\mathrm{s}, 2 \mathrm{H}, \mathrm{CH}_{2}\right), 3.55\left(\mathrm{~s}, 2 \mathrm{H}, \mathrm{CH}_{2}\right) ;{ }^{13} \mathrm{C} \mathrm{NMR}\left(\mathrm{CDCl}_{3}, 100\right.$ MHz) $\delta: 173.46,158.05,141.75,134.35,127.80,126.73$, 126.24, 125.01, 123.83, 108.88, 97.51, 94.6, 94.31, 42.79, 34.62; FT-IR (KBr) $v$ : $1709 \mathrm{~cm}^{-1}$; ESI-MS m/z: 258.0 $[\mathrm{M}+\mathrm{H}]^{+}$. Anal. calcd for $\mathrm{C}_{15} \mathrm{H}_{12} \mathrm{ClNO}$ : C 69.91, H 4.69, N 5.43; found C 69.98, H 4.74, N 5.23.

1-对氯茮基-5-氯吲哚酤 $(\mathbf{5} \mathbf{j})$ : 白色粉末, 产率 83.3\%. m.p. $161.3 \sim 161.8{ }^{\circ} \mathrm{C} ;{ }^{1} \mathrm{H}$ NMR $\left(\mathrm{CDCl}_{3}, 400\right.$ MHz) $\delta: 7.32 \sim 7.08(\mathrm{~m}, 6 \mathrm{H}, \mathrm{ArH}), 6.54(\mathrm{~d}, J=8.3 \mathrm{~Hz}, 1 \mathrm{H}$, $\mathrm{ArH}), 4.80\left(\mathrm{~s}, 2 \mathrm{H}, \mathrm{CH}_{2}\right), 3.52\left(\mathrm{~s}, 2 \mathrm{H}, \mathrm{CH}_{2}\right) ;{ }^{13} \mathrm{C} \mathrm{NMR}$ $\left(\mathrm{CDCl}_{3}, 100 \mathrm{MHz}\right) \delta: 174.46,142.78,135.38,128.82$, 127.74, 127.26, 126.03, 124.85, 109.96, 109.89, 43.81, 35.63; FT-IR (KBr) v: $1703 \mathrm{~cm}^{-1}$; ESI-MS m/z: 293.0 $[\mathrm{M}+\mathrm{H}]^{+}$. Anal. calcd for $\mathrm{C}_{15} \mathrm{H}_{11} \mathrm{Cl}_{2} \mathrm{NO}$ : C 61.67, H 3.79, $\mathrm{N}$ 4.79; found C 61.77, H 3.85, N 4.59.

1-甲基-5-溴吲哚酮 $(5 \mathbf{k})$ : 白色晶体，产率 $88.7 \%$. m.p. 134.4 $134.5{ }^{\circ} \mathrm{C}$ (文献值 ${ }^{[19]}$ : 产率 95.0\%); ${ }^{1} \mathrm{H} \mathrm{NMR}$ $\left(\mathrm{CDCl}_{3}, 400 \mathrm{MHz}\right) \delta: 7.38 \sim 7.25(\mathrm{~m}, 2 \mathrm{H}, \mathrm{ArH}), 6.62$ (d, $J=8.1 \mathrm{~Hz}, 1 \mathrm{H}, \mathrm{ArH}), 3.45\left(\mathrm{~s}, 2 \mathrm{H}, \mathrm{CH}_{2}\right), 3.12\left(\mathrm{~s}, 3 \mathrm{H}, \mathrm{CH}_{3}\right)$; ${ }^{13} \mathrm{C} \mathrm{NMR}\left(\mathrm{CDCl}_{3}, 100 \mathrm{MHz}\right) \delta: 172.49,142.38,128.88$, 125.63, 124.58, 113.09, 107.56, 33.70, 24.44; FT-IR (KBr) $v: 1711 \mathrm{~cm}^{-1}$; ESI-MS m/z: $227.0[\mathrm{M}+\mathrm{H}]^{+}$. Anal. calcd for $\mathrm{C}_{9} \mathrm{H}_{8} \mathrm{BrNO}$ : C 47.82, H 3.57, N 6.20; found C 47.87, $\mathrm{H}$ 3.68, N 6.04 .

1-乙基-5-溴吲哚酮(5I): 淡黄色固体，产率 85.2\%. m.p. $109.4 \sim 110.7{ }^{\circ} \mathrm{C}$ (文献值 ${ }^{[17]}$ : 产率 $59.0 \%$ ); ${ }^{1} \mathrm{H} \mathrm{NMR}$ $\left(\mathrm{CDCl}_{3}, 400 \mathrm{MHz}\right) \delta: 7.20 \sim 7.38(\mathrm{~m}, 2 \mathrm{H}, \mathrm{ArH}), 6.64(\mathrm{~d}$, $J=8.2 \mathrm{~Hz}, 1 \mathrm{H}, \mathrm{ArH}), 3.67$ (dd, $J=7.1,14.3 \mathrm{~Hz}, 2 \mathrm{H}, \mathrm{CH}_{2}$ ), $3.44\left(\mathrm{~s}, 2 \mathrm{H}, \mathrm{CH}_{2}\right), 1.18(\mathrm{t}, J=7.1 \mathrm{~Hz}, 3 \mathrm{H}) ;{ }^{13} \mathrm{C} \mathrm{NMR}$ $\left(\mathrm{CDCl}_{3}, 100 \mathrm{MHz}\right) \delta: 172.10,141.47,128.79,125.85$, 124.88, 112.83, 107.65, 33.79, 32.92, 10.73; FT-IR (KBr) v: $1705 \mathrm{~cm}^{-1}$; ESI-MS m/z: $241.0[\mathrm{M}+\mathrm{H}]^{+}$. Anal. calcd for $\mathrm{C}_{10} \mathrm{H}_{10}$ BrNO: C 50.02, H 4.20, N 5.83; found C 50.12, H 4.27, N 5.64.

1-正丁基-5-溴吲哚酮 $(\mathbf{5 m})$ : 淡黄色晶体，产率 86.0\%. m.p. 58.1 58.2 ${ }^{\circ} \mathrm{C} ;{ }^{1} \mathrm{H} \mathrm{NMR}\left(\mathrm{CDCl}_{3}, 400 \mathrm{MHz}\right)$ $\delta: 7.30(\mathrm{~d}, J=12.7 \mathrm{~Hz}, 2 \mathrm{H}, \mathrm{ArH}), 6.63(\mathrm{~d}, J=8.2 \mathrm{~Hz}, 1 \mathrm{H}$, ArH), 3.60 (t, J=7.3 Hz, 2H, $\mathrm{CH}_{2}$ ), 3.44 (s, 2H, $\mathrm{CH}_{2}$ ), $1.49 \sim 1.60\left(\mathrm{~m}, 2 \mathrm{H}, \mathrm{CH}_{2}\right), 1.23 \sim 1.35(\mathrm{~m}, 2 \mathrm{H}), 0.87(\mathrm{t}, J=$ $\left.7.3 \mathrm{~Hz}, 3 \mathrm{H}, \mathrm{CH}_{3}\right) ;{ }^{13} \mathrm{C} \mathrm{NMR}\left(\mathrm{CDCl}_{3}, 100 \mathrm{MHz}\right) \delta: 172.38$, $141.86,128.75,125.79,124.82,112.85,107.81,38.04$, 33.72, 27.54, 18.34, 11.92; FT-IR (KBr) v: $1703 \mathrm{~cm}^{-1}$; ESI-MS $m / z: 267.0[\mathrm{M}+\mathrm{H}]^{+}$. Anal. calcd for $\mathrm{C}_{12} \mathrm{H}_{14} \mathrm{BrNO}$ : C 53.75, H 5.26, N 5.22; found C 53.86, H 5.35, N 5.25.

1-苠基-5-溴吲哚酩(5n): 米白色粉末，产率 80.5\%. m.p. $125.9 \sim 126.7{ }^{\circ} \mathrm{C} ;{ }^{1} \mathrm{H}$ NMR $\left(\mathrm{CDCl}_{3}, 400 \mathrm{MHz}\right) \delta$ : $7.10 \sim 7.38(\mathrm{~m}, 7 \mathrm{H}, \mathrm{ArH}), 6.49(\mathrm{~d}, J=8.3 \mathrm{~Hz}, 1 \mathrm{H}, \mathrm{ArH})$, $4.81\left(\mathrm{~s}, 2 \mathrm{H}, \mathrm{CH}_{2}\right), 3.53\left(\mathrm{~s}, 2 \mathrm{H}, \mathrm{CH}_{2}\right) ;{ }^{13} \mathrm{C} \mathrm{NMR}\left(\mathrm{CDCl}_{3}\right.$, $100 \mathrm{MHz}) \delta: 172.53,141.44,133.53,128.82,127.01$, $125.95,125.80,125.45,124.62,113.21,108.60,41.96$, 33.72; FT-IR (KBr) v: $1702 \mathrm{~cm}^{-1}$; ESI-MS m/z: 303.0 $[\mathrm{M}+\mathrm{H}]^{+}$. Anal. calcd for $\mathrm{C}_{15} \mathrm{H}_{12} \mathrm{BrNO}$ : C 59.62, $\mathrm{H} 4.00, \mathrm{~N}$ 4.64; found C 59.74, H 4.12, N 4.26.

1-对氯苄基-5-溴吲哚酮 $(50)$ : 淡黄色晶体, 产率 83.2\%. m.p. $185.9 \sim 187.2{ }^{\circ} \mathrm{C} ;{ }^{1} \mathrm{H}$ NMR $\left(\mathrm{CDCl}_{3}, 400\right.$ $\mathrm{MHz}) \delta: 7.30$ (s, 1H, ArH), $7.20(\mathrm{~s}, 3 \mathrm{H}, \mathrm{ArH}), 7.15$ (s, 2H, $\operatorname{ArH}), 6.47$ (d, J=8.1 Hz, 1H, ArH), 4.78 (s, 2H, $\mathrm{CH}_{2}$ ), $3.55\left(\mathrm{~s}, 2 \mathrm{H}, \mathrm{CH}_{2}\right) ;{ }^{13} \mathrm{C} \mathrm{NMR}\left(\mathrm{CDCl}_{3}, 100 \mathrm{MHz}\right) \delta: 172.50$, $141.13,132.05,131.82,128.88,127.20,126.84,125.94$, 125.88, 113.40, 108.44, 108.38, 41.33, 33.67; FT-IR (KBr) v: $1707 \mathrm{~cm}^{-1}$; ESI-MS m/z: $337.0[\mathrm{M}+\mathrm{H}]^{+}$. Anal. calcd for $\mathrm{C}_{15} \mathrm{H}_{11}$ BrClNO: $\mathrm{C}$ 53.52, $\mathrm{H}$ 3.29, $\mathrm{N} 4.16$; found $\mathrm{C}$ 53.62, H 3.33, N 4.01.

\section{$1.5 \mathrm{~N}$-烃基卤代吲哚酮乙酰化反应}

将 $N$-烃基-5-卤代吲哚酮(1 mmol)溶于乙酸䣶(1.5 $\mathrm{mL})$ 中，加入 4-二甲氨基吡啶(DMAP) (0.01 mmol), 反 应结束将反应混合物冷却至室温后加水, 析出的沉淀经 
抽滤、干燥得到酰化产物, 然后溶于四氢呋喃 $(5 \mathrm{~mL})$ 中, 加入质量分数为 $5 \%$ 的 $\mathrm{NaOH}$ 溶液 $(5 \mathrm{~mL})$, 水解反应 12 $\mathrm{h}$, 反应结束后倒入冰水中, 调节至酸性在其过程中溶 液由红色逐渐变为灰白色沉淀, 抽滤, 干燥的粗产品, 经柱层析分离得分析纯产物 3-乙酰基-1-烃基-2-羊基-5卤代吲哚衍生物.

3-乙酰基-1-甲基-2-羟基-5-氟吲哚(6a): 黄棕色粉 末, 产率 65\%. m.p. 141.1 141.9 ${ }^{\circ} \mathrm{C} ;{ }^{1} \mathrm{H}$ NMR $\left(\mathrm{CDCl}_{3}\right.$, $400 \mathrm{MHz}) \delta$ : $13.68(\mathrm{~s}, 1 \mathrm{H}, \mathrm{OH}), 7.00(\mathrm{~d}, J=9.0 \mathrm{~Hz}, 1 \mathrm{H}$, ArH), 6.85 (t, $J=8.9 \mathrm{~Hz}, 1 \mathrm{H}, \mathrm{ArH}), 6.77$ (dd, $J=4.4,8.5$ $\mathrm{Hz}, 1 \mathrm{H}, \mathrm{ArH}), 3.26\left(\mathrm{~s}, 3 \mathrm{H}, \mathrm{CH}_{3}\right), 2.36\left(\mathrm{~s}, 3 \mathrm{H}, \mathrm{CH}_{3}\right) ;{ }^{13} \mathrm{C}$ NMR $\left(\mathrm{CDCl}_{3}, 100 \mathrm{MHz},\right) \delta: 170.95,160.26,157.90$, $111.45,111.21,108.66,108.57,107.33,107.07,25.80$, 20.28; FT-IR (KBr) v: 3072, 2935, $1663 \mathrm{~cm}^{-1}$; ESI-MS $m / z: 208.0[\mathrm{M}+\mathrm{H}]^{+}$. Anal. calcd for $\mathrm{C}_{11} \mathrm{H}_{10} \mathrm{FNO}_{2}: \mathrm{C} 63.76$, H 4.86, N 6.76; found C 63.80, H 4.89, N 6.64.

3-乙酰基-1-乙基-2-羟基-5-氟吲哚(6b): 红色固体: 产率 57\%. m.p 93.9 94.5 ${ }^{\circ} \mathrm{C} ;{ }^{1} \mathrm{H}$ NMR $\left(\mathrm{CDCl}_{3}, 400\right.$ MHz,) $\delta: 13.57$ (s, 1H, OH), 7.05 (d, $J=9.1 \mathrm{~Hz}, 1 \mathrm{H}, \mathrm{ArH}$ ), $6.92 \sim 6.81(\mathrm{~m}, 2 \mathrm{H}, \mathrm{ArH}), 3.86\left(\mathrm{q}, J=7.3 \mathrm{~Hz}, 2 \mathrm{H}, \mathrm{CH}_{2}\right)$, $2.40\left(\mathrm{~d}, J=1.8 \mathrm{~Hz}, 3 \mathrm{H}, \mathrm{CH}_{3}\right), 1.28(\mathrm{t}, J=6.4 \mathrm{~Hz}, 3 \mathrm{H}$, $\left.\mathrm{CH}_{3}\right) ;{ }^{13} \mathrm{C}$ NMR $\left(\mathrm{CDCl}_{3}, 100 \mathrm{MHz}\right) \delta: 174.25,160.10$, 157.74, 111.35, 111.11, 108.77, 108.69, 107.42, 107.16, 34.40, 20.31, 13.19; FT-IR (KBr) v: 3100, 2976, 1664 $\mathrm{cm}^{-1}$; ESI-MS $m / z$ : $222.0[\mathrm{M}+\mathrm{H}]^{+}$. Anal. calcd for $\mathrm{C}_{12} \mathrm{H}_{12} \mathrm{FNO}_{2}$ : C 65.15, H 5.47, N 6.33; found C 65.23, H $5.54, \mathrm{~N} 6.43$.

3-乙酰基-1-正丁基-2-青基-5-氟吲哚(6c): 紫红色晶 体, 产率 $42 \%$. m.p. $79.4 \sim 81.2{ }^{\circ} \mathrm{C} ;{ }^{1} \mathrm{H} \mathrm{NMR}\left(\mathrm{CDCl}_{3}\right.$, $400 \mathrm{MHz}$,$) : 13.69$ (s, 1H, OH), 7.00 (d, $J=9.1 \mathrm{~Hz}, 1 \mathrm{H}$, $\mathrm{ArH}), 6.68 \sim 6.92(\mathrm{~m}, 2 \mathrm{H}, \mathrm{ArH}), 3.74(\mathrm{t}, J=7.1 \mathrm{~Hz}, 2 \mathrm{H}$, $\left.\mathrm{CH}_{2}\right), 2.35\left(\mathrm{~s}, 3 \mathrm{H}, \mathrm{CH}_{3}\right), 1.55 \sim 1.68\left(\mathrm{~m}, 2 \mathrm{H}, \mathrm{CH}_{2}\right), 1.30$ (dd, $\left.J=7.4,14.7 \mathrm{~Hz}, 2 \mathrm{H}, \mathrm{CH}_{2}\right), 0.88(\mathrm{t}, J=7.3 \mathrm{~Hz}, 3 \mathrm{H}$, $\left.\mathrm{CH}_{3}\right) ;{ }^{13} \mathrm{C}$ NMR $\left(\mathrm{CDCl}_{3}, 100 \mathrm{MHz}\right) \delta: 174.29,160.09$, 157.73, 111.32, 111.08, 108.96, 108.87, 107.35, 107.09, 39.50, 29.98, 20.34, 20.15, 13.72; FT-IR (KBr) v: 3066, 2960, $1654 \mathrm{~cm}^{-1}$; ESI-MS m/z: $250.0[\mathrm{M}+\mathrm{H}]^{+}$. Anal. calcd for $\mathrm{C}_{14} \mathrm{H}_{16} \mathrm{FNO}_{2}$ : C 67.45, H 6.47, N 5.62; found $\mathrm{C}$ 67.53, H 6.57, N 5.53 .

3-乙酰基-1-芐基-2-羊基-5-氟吲哚(6d): 紫色固体, 产率 67\%. m.p. 101.0 102.3 ${ }^{\circ} \mathrm{C} ;{ }^{1} \mathrm{H}$ NMR (DMSO- $d_{6}$, $400 \mathrm{MHz}) \delta$ : 12.15 (s, 1H, OH), $7.46(\mathrm{~d}, J=8.1 \mathrm{~Hz}, 1 \mathrm{H}$, ArH), 7.28 (td, $J=7.3,14.5 \mathrm{~Hz}, 5 \mathrm{H}, \mathrm{ArH}), 6.83$ (d, $J=7.8$ $\mathrm{Hz}, 2 \mathrm{H}, \mathrm{ArH}), 4.95\left(\mathrm{~s}, 2 \mathrm{H}, \mathrm{CH}_{2}\right), 2.64\left(\mathrm{~s}, 3 \mathrm{H}, \mathrm{CH}_{3}\right) ;{ }^{13} \mathrm{C}$ NMR (DMSO- $\left.d_{6}, 100 \mathrm{MHz}\right) \delta: 172.85,159.56,157.26$,
137.81, 134.16, 128.97, 127.76, 127.67, 124.61, 110.78, 109.01, 108.35, 42.65, 19.58; FT-IR (KBr) v: 3030, 2976, $1664 \mathrm{~cm}^{-1}$; ESI-MS $m / z: 284.0[\mathrm{M}+\mathrm{H}]^{+}$. Anal. calcd for $\mathrm{C}_{17} \mathrm{H}_{14} \mathrm{FNO}_{2}$ : C 72.07, $\mathrm{H} 4.98, \mathrm{~N}$ 4.94; found $\mathrm{C} 72.15, \mathrm{H}$ $5.04, \mathrm{~N} 4.86$.

3-乙酰基-1-对氯芐基-2-羊基-5-氟吲哚(6e): 紫色 粉, 产率 80\%. m.p. 291.4 292.6 ${ }^{\circ} \mathrm{C}$; ${ }^{1} \mathrm{H}$ NMR (DMSO$\left.d_{6}, 400 \mathrm{MHz}\right) \delta: 12.10(\mathrm{~s}, 1 \mathrm{H}, \mathrm{OH}), 7.49$ (s, $\left.1 \mathrm{H}, \mathrm{ArH}\right), 7.42$ (s, 2H, ArH), 7.33 (s, 2H, ArH), 6.88 (s, 2H, ArH), 4.99 (s, $2 \mathrm{H}, \mathrm{CH}_{2}$ ), 2.67 (s, $3 \mathrm{H}, \mathrm{CH}_{3}$ ); ${ }^{13} \mathrm{C}$ NMR (DMSO- $d_{6}, 100$ MHz) $\delta: 180.25,159.63,156.41,136.81,133.94,132.12$, $129.49,128.95,110.79,109.98,109.25,108.26,41.98$, 19.25; FT-IR (KBr) $v: 3089,2950,1662 \mathrm{~cm}^{-1}$; ESI-MS $m / z: 318.0[\mathrm{M}+\mathrm{H}]^{+}$. Anal. calcd for $\mathrm{C}_{17} \mathrm{H}_{13} \mathrm{ClFNO}_{2}$ : C 64.26, H 4.12, N 4.41; found C 64.36, H 4.25, N 4.53.

3-乙酰基-1-甲基-2-羊基-5-氯吲哚(6f): 紫白色晶体, 产率 45\%. m.p. $119.0 \sim 120.8{ }^{\circ} \mathrm{C} ;{ }^{1} \mathrm{H}$ NMR $\left(\mathrm{CDCl}_{3}, 400\right.$ $\mathrm{MHz},) \delta: 13.52(\mathrm{~s}, 1 \mathrm{H}, \mathrm{OH}), 7.24(\mathrm{~s}, 1 \mathrm{H}, \mathrm{ArH}), 7.11(\mathrm{~d}$, $J=8.1 \mathrm{~Hz}, 1 \mathrm{H}, \mathrm{ArH}), 6.78(\mathrm{~d}, J=8.3 \mathrm{~Hz}, 1 \mathrm{H}, \mathrm{ArH}), 3.26$ (s, 3H, $\left.\mathrm{CH}_{3}\right), 2.38\left(\mathrm{~s}, 3 \mathrm{H}, \mathrm{CH}_{3}\right) ;{ }^{13} \mathrm{C}$ NMR $\left(\mathrm{CDCl}_{3}, 100\right.$ MHz) $\delta: 174.22,170.80,137.18,127.53,124.83,123.58$, 119.68, 109.12, 102.47, 101.18, 25.79, 20.42; FT-IR (KBr) v: 2955, $1670 \mathrm{~cm}^{-1}$; ESI-MS m/z: $224.0[\mathrm{M}+\mathrm{H}]^{+}$. Anal. calcd for $\mathrm{C}_{11} \mathrm{H}_{10} \mathrm{ClNO}_{2}$ : C 59.07, H 4.51, N 6.26; found C 59.16, H 4.58, N 6.37.

3-乙酰基-1-乙基-2-羟基-5-氯吲哚(6g): 紫色晶体, 产率 35\%. m.p. $77.7 \sim 78.9{ }^{\circ} \mathrm{C} ;{ }^{1} \mathrm{H}$ NMR $\left(\mathrm{CDCl}_{3}, 400\right.$ $\mathrm{MHz}$ ) $\delta: 13.73$ (s, 1H, OH), 7.30 (s, 1H, ArH), $7.15(\mathrm{~d}$, $J=8.3 \mathrm{~Hz}, 1 \mathrm{H}, \mathrm{ArH}), 6.86(\mathrm{~d}, J=8.1 \mathrm{~Hz}, 1 \mathrm{H}, \mathrm{ArH}), 3.86$ (dd, $\left.J=13.6,6.6 \mathrm{~Hz}, 2 \mathrm{H}, \mathrm{CH}_{2}\right), 2.43\left(\mathrm{~s}, 3 \mathrm{H}, \mathrm{CH}_{3}\right), 1.28(\mathrm{t}$, $\left.J=6.6 \mathrm{~Hz}, 3 \mathrm{H}, \mathrm{CH}_{3}\right) ;{ }^{13} \mathrm{C} \mathrm{NMR}\left(\mathrm{CDCl}_{3}, 100 \mathrm{MHz}\right) \delta$ : $174.10,170.53,136.13,127.32,124.73,123.85,119.87$, 109.24, 101.24, 34.43, 20.47, 13.21; FT-IR (KBr) v: 1979, $1688 \mathrm{~cm}^{-1}$; ESI-MS $m / z$ : $238.0[\mathrm{M}+\mathrm{H}]^{+}$. Anal. calcd for $\mathrm{C}_{12} \mathrm{H}_{12} \mathrm{ClNO}_{2}$ : C 60.64, H 5.09, N 5.89; found C 60.75, H $5.28, \mathrm{~N} 5.68$.

3-乙酰基-1-正丁基-2-羊基-5-氯吲梠(6h): 紫色固 体, 产率 32\%. m.p. $73.3 \sim 75.1{ }^{\circ} \mathrm{C} ;{ }^{1} \mathrm{H}$ NMR $\left(\mathrm{CDCl}_{3}\right.$, $400 \mathrm{MHz}) \delta: 13.64(\mathrm{~s}, 1 \mathrm{H}, \mathrm{OH}), 7.21(\mathrm{~s}, 1 \mathrm{H}, \mathrm{ArH}), 7.07$ (d, $J=8.3 \mathrm{~Hz}, 1 \mathrm{H}, \mathrm{ArH}), 6.77$ (d, $J=8.4 \mathrm{~Hz}, 1 \mathrm{H}, \mathrm{ArH}), 3.72$ (t, $\left.J=7.0 \mathrm{~Hz}, 2 \mathrm{H}, \mathrm{CH}_{2}\right), 2.35$ (s, $\left.3 \mathrm{H}, \mathrm{CH}_{3}\right), 1.59$ (dt, $J=$ $\left.6.4,12.8 \mathrm{~Hz}, 2 \mathrm{H}, \mathrm{CH}_{2}\right), 1.25 \sim 1.31\left(\mathrm{~m}, 2 \mathrm{H}, \mathrm{CH}_{2}\right), 0.86$ (t, $\left.J=7.3 \mathrm{~Hz}, 3 \mathrm{H}, \mathrm{CH}_{3}\right) ;{ }^{13} \mathrm{C} \mathrm{NMR}\left(\mathrm{CDCl}_{3}, 100 \mathrm{MHz}\right) \delta$ : $174.39,170.71,136.48,127.26,124.66,123.76,119.74$, 109.40, 101.06, 39.48, 29.97, 20.46, 20.13, 13.71; FT-IR 
(KBr) $v$ : 2955, $1654 \mathrm{~cm}^{-1}$; ESI-MS $m / z: 266.0[\mathrm{M}+\mathrm{H}]^{+}$. Anal. calcd for $\mathrm{C}_{14} \mathrm{H}_{16} \mathrm{ClNO}_{2}$ : C 63.28, $\mathrm{H}$ 6.07, N 5.27; found C 63.35, H 6.28, N 5.12.

3-乙酰基-1-芐基-2-羊基-5-氯吲哚(6i): 紫红色粉末, 产率 40\%. m.p. $>300{ }^{\circ} \mathrm{C} ;{ }^{1} \mathrm{H}$ NMR (DMSO- $d_{6}, 400 \mathrm{MHz}$ ) $\delta: 12.46(\mathrm{~s}, 1 \mathrm{H}, \mathrm{OH}), 7.66(\mathrm{~s}, 1 \mathrm{H}, \mathrm{ArH}), 7.33 \sim 7.21(\mathrm{~m}$, $5 \mathrm{H}, \mathrm{ArH}), 7.08(\mathrm{~s}, 1 \mathrm{H}, \mathrm{ArH}), 6.85(\mathrm{~s}, 1 \mathrm{H}, \mathrm{ArH}), 4.95(\mathrm{~s}$, $2 \mathrm{H}, \mathrm{CH}_{2}$ ), 2.65 (s, $3 \mathrm{H}, \mathrm{CH}_{3}$ ); ${ }^{13} \mathrm{C}$ NMR (DMSO- $d_{6}, 100$ $\mathrm{MHz}$,$) : 172.48,148.24,137.62,136.76,128.99,128.34$, $127.54,125.81,124.70,121.70,109.25,101.35,42.61$, 18.95; FT-IR (KBr) v: 2924, $1618 \mathrm{~cm}^{-1}$; ESI-MS $m / z$ : $300.0[\mathrm{M}+\mathrm{H}]^{+}$. Anal. calcd for $\mathrm{C}_{17} \mathrm{H}_{14} \mathrm{ClNO}_{2}: \mathrm{C} 68.12, \mathrm{H}$ 4.71, N 4.67; found C 68.22, H 4.61, N 4.51.

3-乙酰基-1-对氯芐基-2-羟基-5-氯吲哚(6j): 紫色固 体, 产率 38\%. m.p. 285.2 285.6 ${ }^{\circ} \mathrm{C}$; ${ }^{1} \mathrm{H}$ NMR (DMSO$\left.d_{6}, 400 \mathrm{MHz}\right) \delta: 12.54$ (s, 1H, OH), 7.66 (s, 2H, ArH), 7.33 (s, 2H, ArH), 7.28 (s, H, ArH) $7.08(\mathrm{~s}, 1 \mathrm{H}, \mathrm{ArH}), 6.84(\mathrm{~s}$, 1H, $\mathrm{ArH}$ ), 4.95 (s, 2H, $\mathrm{CH}_{2}$ ), 2.64 (s, 3H, $\mathrm{CH}_{3}$ ); ${ }^{13} \mathrm{C} \mathrm{NMR}$ (DMSO- $\left.d_{6}, 100 \mathrm{MHz}\right) \delta: 172.69,136.68,136.54,132.22$, 129.46, 129.11, 128.98, 124.72, 121.73, 111.09, 109.83, 109.29, 41.96, 19.31; FT-IR (KBr) v: 2934, $1610 \mathrm{~cm}^{-1}$; ESI-MS m/z: $335.0[\mathrm{M}+\mathrm{H}]^{+}$. Anal. calcd for $\mathrm{C}_{17} \mathrm{H}_{13} \mathrm{Cl}_{2}-$ $\mathrm{NO}_{2}$ : C 61.10, H 3.92, N 4.19; found C 61.24, H 4.03, N 4.02 .

3-乙酰基-1-甲基-2-羟基-5-溴吲梠(6k): 紫色晶体, 产率 58\%. m.p. 132.0 134.1 ${ }^{\circ} \mathrm{C} ;{ }^{1} \mathrm{H}$ NMR $\left(\mathrm{CDCl}_{3}, 400\right.$ MHz) $\delta: 13.63(\mathrm{~s}, 1 \mathrm{H}, \mathrm{OH}), 7.36(\mathrm{~s}, 1 \mathrm{H}, \operatorname{ArH}), 7.25(\mathrm{~d}$, $J=8.0 \mathrm{~Hz}, 1 \mathrm{H}, \mathrm{ArH}), 6.73(\mathrm{~d}, J=8.2 \mathrm{~Hz}, 1 \mathrm{H}, \mathrm{ArH}), 3.25$ (s, $\left.3 \mathrm{H}, \mathrm{CH}_{3}\right), 2.37\left(\mathrm{~s}, 3 \mathrm{H}, \mathrm{CH}_{3}\right) ;{ }^{13} \mathrm{C}$ NMR $\left(\mathrm{CDCl}_{3}, 100\right.$ MHz) $\delta: 173.29,136.55,129.88,127.79,126.64,123.03$, 121.42, 113.94, 108.61, 24.73, 19.44; FT-IR (KBr) v: 2940, $1617 \mathrm{~cm}^{-1}$; ESI-MS m/z: $269.0[\mathrm{M}+\mathrm{H}]^{+}$. Anal. calcd for $\mathrm{C}_{11} \mathrm{H}_{10} \mathrm{BrNO}_{2}$ : C 49.28, H 3.76, N 5.22; found C 49.37, H 3.86, N 5.03.

3-乙酰基-1-乙基-2-羟基-5-溴吲哚(61): 紫色粉末, 产率 56\%. m.p. 86.9 87.1 ${ }^{\circ} \mathrm{C} ;{ }^{1} \mathrm{H}$ NMR $\left(\mathrm{CDCl}_{3}, 400\right.$ MHz) $\delta$ : 13.67 (s, 1H, OH), 7.39 (s, 1H, ArH), 7.24 (d, $J=8.3 \mathrm{~Hz}, 1 \mathrm{H}, \mathrm{ArH}), 6.77$ (d, $J=8.3 \mathrm{~Hz}, 1 \mathrm{H}, \mathrm{ArH}), 3.80$ (d, $J=7.2 \mathrm{~Hz}, 2 \mathrm{H}, \mathrm{CH}_{2}$ ), 2.37 (s, 3H, $\left.\mathrm{CH}_{3}\right), 1.22$ (t, $J=7.2$ $\left.\mathrm{Hz}, 3 \mathrm{H}, \mathrm{CH}_{3}\right) ;{ }^{13} \mathrm{C}$ NMR $\left(\mathrm{CDCl}_{3}, 100 \mathrm{MHz}\right) \delta: 174.43$, $136.56,136.56,127.58,124.31,122.60,114.73,109.96$, 109.75, 101.07, 34.41, 20.52, 13.18; FT-IR (KBr) v: 2932, $1615 \mathrm{~cm}^{-1}$; ESI-MS $m / z: 283.0[\mathrm{M}+\mathrm{H}]^{+}$. Anal. calcd for $\mathrm{C}_{12} \mathrm{H}_{12} \mathrm{BrNO}_{2}$ : C 51.09, H 4.29, N 4.96; found C 51.19, H 4.41, N 4.78. 3-乙酰基-1-正丁基-2-羟基-5-溴吲哚(6m): 紫色晶 体, 产率 48\%. m.p. 57.7 58.9 ${ }^{\circ} \mathrm{C} ;{ }^{1} \mathrm{H}$ NMR $\left(\mathrm{CDCl}_{3}, 400\right.$ MHz) $\delta: 13.75$ (s, 1H, OH), 7.39 (s, 1H, ArH), 7.21 (s, 1H, $\mathrm{ArH}), 6.77$ (s, $1 \mathrm{H}, \mathrm{ArH}), 3.76\left(\mathrm{~s}, 2 \mathrm{H}, \mathrm{CH}_{2}\right), 2.38(\mathrm{~s}, 2 \mathrm{H}$, $\left.\mathrm{CH}_{2}\right), 1.61\left(\mathrm{~s}, 2 \mathrm{H}, \mathrm{CH}_{2}\right), 1.38 \sim 1.05\left(\mathrm{~s}, 3 \mathrm{H}, \mathrm{CH}_{3}\right), 0.83(\mathrm{~s}$, $\left.3 \mathrm{H}, \mathrm{CH}_{3}\right) ;{ }^{13} \mathrm{C} \mathrm{NMR}\left(\mathrm{CDCl}_{3}, 100 \mathrm{MHz}\right) \delta: 174.53,136.92$, $127.54,124.45,124.29,122.55,114.72,109.96,101.15$, 39.47, 29.98, 20.55, 20.18, 13.72; FT-IR (KBr) v: 2948, $1621 \mathrm{~cm}^{-1}$; ESI-MS $m / z: 311.0[\mathrm{M}+\mathrm{H}]^{+}$. Anal. calcd for $\mathrm{C}_{14} \mathrm{H}_{16} \mathrm{BrNO}_{2}$ : C 54.21, H 5.20, N 4.52; found C 54.43, H $5.35, \mathrm{~N} 4.38$.

3-乙酰基-1-芐基-2-羟基-5-溴吲哚(6n): 紫色固体, 产率 51\%. m.p. $175.8 \sim 177.2{ }^{\circ} \mathrm{C}$; $1 \mathrm{H}$ NMR (DMSO- $d_{6}$, $400 \mathrm{MHz}) \delta: 12.47(\mathrm{~s}, 1 \mathrm{H}, \mathrm{OH}), 7.79(\mathrm{~s}, 1 \mathrm{H}, \mathrm{ArH}), 7.38(\mathrm{~d}$, $J=6.7 \mathrm{~Hz}, 2 \mathrm{H}, \mathrm{ArH}), 7.31 \sim 7.15(\mathrm{~m}, 3 \mathrm{H}, \mathrm{ArH}), 6.82(\mathrm{~s}$, $1 \mathrm{H}, \mathrm{ArH}), 4.98\left(\mathrm{~s}, 2 \mathrm{H}, \mathrm{CH}_{2}\right), 2.64\left(\mathrm{~s}, 3 \mathrm{H}, \mathrm{CH}_{3}\right) ;{ }^{13} \mathrm{C} \mathrm{NMR}$ (DMSO- $\left.d_{6}, 100 \mathrm{MHz}\right) \delta: 172.85,165.15,143.79,136.90$, $132.23,129.45,128.98,127.56,124.40,119.14,113.62$, 109.98, 104.64, 101.15, 41.93, 19.00; FT-IR (KBr) $v$ : 2962, $1638 \mathrm{~cm}^{-1}$; ESI-MS m/z: $345.0[\mathrm{M}+\mathrm{H}]^{+}$. Anal. calcd for $\mathrm{C}_{17} \mathrm{H}_{14} \mathrm{BrNO}_{2}$ : C 59.32, H 4.10, N 4.07; found $\mathrm{C}$ 59.42, H 4.24, N 3.92.

3-乙酰基-1-对氯苠基-2-羟基-5-溴吲哚(60): 紫色粉 末, 产率 43\%. m.p. 139.1 $140.8{ }^{\circ} \mathrm{C}$; ${ }^{1} \mathrm{H}$ NMR (DMSO$d_{6}, 400 \mathrm{MHz}$ ) $\delta: 12.47(\mathrm{~s}, 1 \mathrm{H}, \mathrm{OH}), 7.80(\mathrm{~s}, 1 \mathrm{H}, \mathrm{ArH})$, $7.48(\mathrm{~d}, J=8.7 \mathrm{~Hz}, 1 \mathrm{H}, \operatorname{ArH}), 7.30(\mathrm{~d}, J=7.0 \mathrm{~Hz}, 2 \mathrm{H}$, $\mathrm{ArH}$ ), 7.26 (s, 3H, $\mathrm{ArH}), 4.95$ (s, 2H, $\mathrm{CH}_{2}$ ), 2.66 (s, 3H, $\mathrm{CH}_{3}$ ); ${ }^{13} \mathrm{C}$ NMR (DMSO- $\left.d_{6}, 100 \mathrm{MHz}\right) \delta: 172.48,169.48$, $149.67,137.59,137.15,130.32,129.86,128.98,127.59$, $125.82,124.44,124.20,113.51,109.83,101.25,42.89$, 21.24; FT-IR (KBr) v: 2957, $1647 \mathrm{~cm}^{-1}$; ESI-MS $\mathrm{m} / z$ : $379.0[\mathrm{M}+\mathrm{H}]^{+}$. Anal. calcd for $\mathrm{C}_{17} \mathrm{H}_{13} \mathrm{BrClNO}_{2}$ : C 53.92, H 3.46, N 3.70; found C 54.02, H 3.46, N 3.56.

\section{2 结果与讨论}

\section{1 卤素功能化靛红的合成}

靛红, 又名吲哚满二酮, 存在于很多植物和海洋生 物当中，是合成颜料、染料以及医药包括消炎镇痛药二 氯芬酸和治疗阿尔茨海默氏病的他克宁等的重要原 料 ${ }^{[16,17]}$. 最近研究表明, 静红还是人类新发现的一种内 源性单胺氧化酶 B (MAO-B)特异抑制因子具有多种生 物活性, 而 MAO-B 是作为参与体内单胺类物质代谢的 主要酶类, 通过对 DA 代谢的抑制作用, 可控制老年性 震颤麻痹 症 ${ }^{[18]}$. 更有文献报道, 靛红具有降低血胆固 醇水平、抗衰老、神经病变等功效 ${ }^{[19,20]}$. 同时靛红也是 
实验室中常用的指示剂 及人体蛋白质测定所需要的重 要试剂[21].

静红的合成主要有以下几种方法: Martinet 合成 法 ${ }^{[22]}$, 以芳香胺和丙酮二酸酯作用, 在有氧的条件下水 解、脱羧; Stoll 合成法 ${ }^{[23]}$, 通过苯胺与草酰氯作用后, Lewis 酸闭环制得靛红, 此方法主要是制备含有多环的 靛红; 还可以对苯胺中的氨基进行叔丁氧羰保护, 然后 与叔丁基锂、草酸二乙酯作用后, 在亲电环境下合成靛 红 ${ }^{[24]}$; Gassman 合成法 ${ }^{[25]}$, 此方法与其它方法有所不同, 苯胺与甲硫基乙酸酯作用先闭环生成 3-甲硫基-2-羟基 吲哚, 再被氧化成靛红, 同时也会生成副产物邻氨基苯 甲酸衍生物. 本研究采用 Sandmeyer 合成法 ${ }^{[26]}$, 与其它 方法相比更具有经济价值, 由于此方法成本低收率高, 且合成条件温和, 是合成靛红的常用方法. 我们通过对 实验条件的改进, 合成出一系列取代的靛红, 与文献 $[27,28]$ 相比, 产率得到一定提高. 其中有文献对甲基异 亚硝基乙酰苯胺的提纯采用先用碱溶解过滤杂质, 再酸 化析出的方法; 文献 ${ }^{[28]}$ 邻乙基异亚硝基乙酰苯胺则采用 乙醚一正己烷重结晶, 考虑到操作较为繁琐, 我们采用水 做重结晶的溶剂, 简单方便, 环境友好, 成本降低.

经 Sandmeyer 靛红合成法, 以对卤代苯胺(1)为底物 与水合氯醛、盐酸着弪胺反应合成取代的异亚硝基乙酰苯 胺, 用浓硫酸使其闭环制得以下三种常用的卤素取代靛 红，其反应方程式如 Scheme 2.

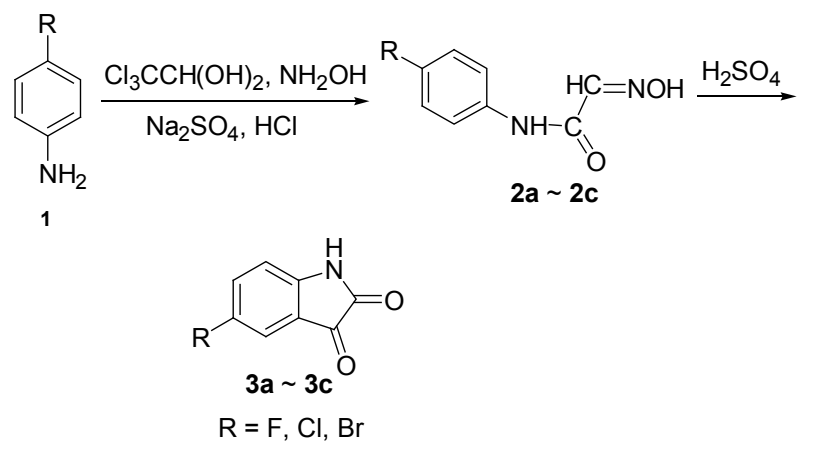

Scheme 2

通过实验发现, 硫酸钠的用量、反应时间和温度对 化合物 $\mathbf{2 a} \sim \mathbf{2 c}$ 的收率影响较大. 硫酸钠可以降低苯胺 的浓度, 适当的增加用量可以减少副产物, 反应温度越 高, 时间会相应缩短, 但副产物增多, 出现大量棕色焦 油状副产物, 不利于产品的提纯; 温度较低, 反应时间 会增长, 但是反应时间过长同样会导致副反应的发生, 影响产率. 综合考虑三个影响因素, 适当升高温度的同 时, 增加硫酸钠的用量, 既缩短反应时间, 又减少了副 物. 通过实验发现, 在 $85{ }^{\circ} \mathrm{C}$ 下反应 $1.5 \sim 2.5 \mathrm{~h}$ 实验条件 最佳. 由于后处理是选用水做重结晶溶剂, 反应结束后
需使反应体系迅速冷却，既可以让产品析出完全，又可 以防止硫酸钠晶体的大量析出而不利于提纯. 按同样的 方法以 $83.4 \% \sim 92.2 \%$ 的收率得到化合物 $\mathbf{2 a} \sim \mathbf{2 c}$ (见表 1).

表 1 化合物 $\mathbf{2 a} \sim \mathbf{2 c}$ 的收率和熔点

Table 1 Yields and melting points of compounds $\mathbf{2 a} \sim \mathbf{2 c}$

\begin{tabular}{cccc}
\hline Compd. & Time $/ \mathrm{h}$ & Yield/\% & m.p. $/{ }^{\circ} \mathrm{C}$ \\
\hline $\mathbf{2 a}$ & 1.5 & 92.5 & $155.3 \sim 156.1$ \\
$\mathbf{2 b}$ & 2.0 & 85.5 & $173.5 \sim 174.6$ \\
$\mathbf{2 c}$ & 2.5 & 83.4 & $168.6 \sim 168.7$ \\
\hline
\end{tabular}

在合成化合物 $3 \mathrm{a} \sim 3 \mathrm{c}$ 的过程当中, 浓硫酸的用量 和反应温度对实验影响较为明显, 浓硫酸的作用是加 成、脱水闭环，用量大时，会导致副反应的发生，用量太 少则反应进行不完全. 产率随着温度的提高而增大, 但 也要有一定的限度，以防止温度过高而碳化，原料必须 完全干燥. 由于加料过程中会放出大量的热, 要保持反 应体系在 $60 \sim 70{ }^{\circ} \mathrm{C}$, 加料速度一定要适中, 不能太快, 要少量多次的加料, 并保持在 $1 \mathrm{~h}$ 之内加完, 否则同样 会降低产率. 按同样的实验条件, 按化合物 $\mathbf{3}$ 计, 以 $80.4 \% \sim 93.5 \%$ 的收率得到化合物 3a $\sim 3 \mathbf{c}$ (见 Table 2).

表 2 化合物 3a $3 \mathrm{c}$ 的收率和熔点

Table 2 Yields and melting points of compounds $\mathbf{3 a} \sim \mathbf{3 c}$

\begin{tabular}{cccc}
\hline Compd. & Time $/ \mathrm{h}$ & Yield $/ \%$ & m.p. $/{ }^{\circ} \mathrm{C}$ \\
\hline 3a & 1.0 & 93.5 & $231.4 \sim 232.8$ \\
3b & 2.0 & 90.7 & $236.7 \sim 239.5$ \\
3c & 2.5 & 80.4 & $260.3 \sim 260.8$ \\
\hline
\end{tabular}

在以上的卤素取代靛红的合成研究中发现，随着取 代基吸电子能力的增强, 其相应靛红的产率越高. 我们 为了证实 $\mathbf{3 a}$ 的结构, 我们对其进行了 ${ }^{1} \mathrm{H} N \mathrm{NR}$ 光谱测定 分析其结构. 在 3a 的 ${ }^{1} \mathrm{H}$ NMR 中 $\delta 11.05$ 处出现的单峰, 归属于靛红上亚氨基上的质子氢. $\delta 7.54 \sim 7.34$ 处出现的 多重峰, 归属于靛红环上的 4 位和 6 位上的质子氢. $\delta$ 6.92 处出现的双峰 $(J=8.6 \mathrm{~Hz})$, 归属于静红上 7 位上的 质子氢.

\section{2 靛红烃基化反应}

氮上烃基化反应的方法有很多主要按照不同的催 化条件分为金属催化 ${ }^{[29]}$ 、分子篮催化 ${ }^{[30]} 、$ 酸催化 ${ }^{[31]}$ 、碱 催化 ${ }^{[32]}$ 四种. 因为前几种的操作条件要求较高而且其成 本较碱催化高. 因而我们实验室按照碱催化的烃基化方 法, 参照文献[33]合成一系列的氮烃基化产物(表 3). 为 了证实 4a 的结构, 我们对其进行了 IR, ${ }^{1} \mathrm{H}$ NMR, ${ }^{13} \mathrm{C}$ NMR 等光谱测定和元素分析. 在 $\mathbf{4 a}$ 的 IR 图中我们发现 在 $1705 \mathrm{~cm}^{-1}$ 处出现的强吸收峰为为 $\mathrm{C}=\mathrm{O}$ 的伸缩振动. ${ }^{1} \mathrm{H}$ NMR 中我们发现在 $\delta 7.03 \sim 7.08$ 处出现的多重峰, 归属于吲哚环上 4 位和 6 位碳上的质子氢, $\delta 6.58 \sim 6.60$ 
处出现的多重峰, 归属于吲哚环上 7 位碳上的质子氢, $\delta$ 2.98 处出现的单重峰, 归属于 1 位氮上甲基上的质子氢. 在 ${ }^{13} \mathrm{C}$ NMR 谱图中 $\delta 170.99,167.97$ 处出现了两个羰基 碳的特征吸收峰. 元素分析数据也与我们推测的化合物 结构相符. 以上数据证实得到的化合物为 1-甲基-5-氟靛 红，化合物 $\mathbf{4 a} \sim \mathbf{4 0}$ 合成路线见 Eq. 1.

表 3 5-卤素取代靛红烃基化的反应结果

Table 3 Results of alkylation of 5-haloindoline-2,3-dione

\begin{tabular}{|c|c|c|c|}
\hline Substrate & Product & Time/h & Yield $^{a} / \%$ \\
\hline $3 \mathbf{a}$ & $4 a$ & 5 & 75.5 \\
\hline $3 \mathbf{a}$ & $4 b$ & 7 & 62.8 \\
\hline $3 \mathbf{a}$ & $4 c$ & 11 & 55.5 \\
\hline $3 \mathbf{a}$ & $4 d$ & 10 & 82.4 \\
\hline $3 \mathbf{a}$ & $4 e$ & 12 & 90.7 \\
\hline $\mathbf{3 b}$ & $4 f$ & 7 & 90.5 \\
\hline $3 b$ & $4 g$ & 8 & 95.4 \\
\hline $3 \mathbf{b}$ & $4 h$ & 10 & 75.5 \\
\hline $3 \mathbf{b}$ & $4 i$ & 12 & 94.1 \\
\hline $3 \mathbf{b}$ & $4 j$ & 14 & 97.5 \\
\hline $3 c$ & $4 k$ & 6 & 90.1 \\
\hline $3 c$ & 41 & 10 & 83.6 \\
\hline $3 c$ & $4 m$ & 11 & 86.7 \\
\hline $3 c$ & $4 n$ & 13 & 90.2 \\
\hline $3 c$ & 40 & 15 & 70.3 \\
\hline
\end{tabular}

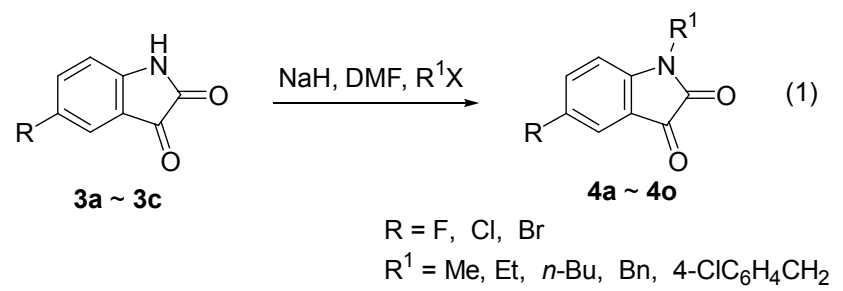

我们研究了三种甾素取代靛红的烃基化反应，在反 应条件相同的条件下，当取代基相同是，如果烃基化基 团越大其反应产率较低, 反应时间较长, 反之则反应时 间短而且反应更完全. 在对比三种靛红在同一种烃基化 试剂作用下, 取代基的电负性越高其反应越完全, 产率 越高, 反应时间也越短. 综合以上的实验数据, 我们了 解到不同卤素取代基和空间位阻对靛红烃基化反应的 影响.

\section{$2.3 N$-烃基化靛红还原反应}

在合成 $5 \mathrm{a} \sim 50$ 时, 我们开始用经典的黄鸣龙还原 反应来合成，但是该方法需要两步才能得到目标产物， 操作比较繁琐, 且总收率较低. 为此, 我们对该方法进 行了改进, 对生成的中间体不进行分离处理, 直接一锅 法合成出 $\mathbf{5 a} \sim \mathbf{5 0}$. 这样不仅简化了实验步骤, 而且收率 较高(表 4). 为了证实 $\mathbf{5 b}$ 的结构, 我们对其进行了 IR, ${ }^{1} \mathrm{H}$ $\mathrm{NMR},{ }^{13} \mathrm{C} \mathrm{NMR}$ 等光谱测定和元素分析. 在 $\mathbf{5 b}$ 的 IR 图
中可见, 在 $1715 \mathrm{~cm}^{-1}$ 处出现的强吸收峰为为 $\mathrm{C}=\mathrm{O}$ 的 伸缩振动. ${ }^{1} \mathrm{H}$ NMR 中 $\delta 6.88 \sim 6.94$ 处出现的重峰, 归属 于吲哚环上 4 位和 6 位碳上的质子氢. $\delta 6.66 \sim 6.69$ 处出 现的多重峰, 归属于吲哚环上 7 位碳上的质子氢, $\delta 3.75$ 处出现的单重峰归属于 1 位氮上烷乙基上的亚甲基的质 子氢. $\delta 1.18$ 处出现的三重峰 $(J=7.2 \mathrm{~Hz})$, 归属于 1 位氮 上烷乙基上甲基的质子氢. 在 ${ }^{13} \mathrm{C} \mathrm{NMR}$ 中 $\delta 173.09$ 处出 现了羰基碳的特征吸收峰. 元素分析数据也与我们推测 的化合物结构相符. 以上数据证实得到的化合物 $\mathbf{5 b}$ 为 1-乙基-5-氟吲哚酮. 化合物 $\mathbf{5 a} \sim \mathbf{5 0}$ 合成路线见 Eq. 2 .

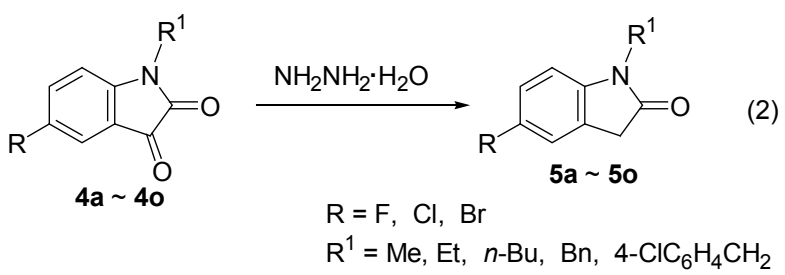

表 4 5-卤素-1-烃基取代靛红还原的反应结果

Table 4 Results of reduction of 5-halo-1-alkylindolin-2,3-dione

\begin{tabular}{ccc}
\hline Substrate & Product & Yield $^{a} / \%$ \\
\hline $\mathbf{4 a}$ & $\mathbf{5 a}$ & 82.5 \\
$\mathbf{4 b}$ & $\mathbf{5 b}$ & 73.2 \\
$\mathbf{4 c}$ & $\mathbf{5 c}$ & 75.6 \\
$\mathbf{4 d}$ & $\mathbf{5 d}$ & 81.2 \\
$\mathbf{4 e}$ & $\mathbf{5 e}$ & 92.6 \\
$\mathbf{4} \mathbf{f}$ & $\mathbf{5 f}$ & 84.0 \\
$\mathbf{4 g}$ & $\mathbf{5 g}$ & 86.1 \\
$\mathbf{4 h}$ & $\mathbf{5 h}$ & 76.2 \\
$\mathbf{4} \mathbf{i}$ & $\mathbf{5 i}$ & 85.1 \\
$\mathbf{4 j}$ & $\mathbf{5 j}$ & 83.3 \\
$\mathbf{4} \mathbf{k}$ & $\mathbf{5 k}$ & 88.7 \\
$\mathbf{4 l}$ & $\mathbf{5 l}$ & 85.2 \\
$\mathbf{4 m}$ & $\mathbf{5 m}$ & 86.0 \\
$\mathbf{4 n}$ & $\mathbf{5 n}$ & 80.5 \\
$\mathbf{4 0}$ & $\mathbf{5 o}$ & 83.2 \\
\hline
\end{tabular}

$a$ 分离产率.

从表 4 中还原产率看出, 苯环上甾素和氮上的烃基 对于还原反应的影响表现的不明显.

\subsection{1-取代吲哚-2-酮乙酰化反应}

按照文献 ${ }^{[34]}$ 合成 3-乙酰基-1-烃基-2-差基吲哚化合 物，以 $N$-烃基卤代吲哚酩(3)为原料，乙酸酐为反应溶剂 在 DMAP 作为酰化催化剂, $150{ }^{\circ} \mathrm{C}$ 反应条件下回流过 夜, 反应结束将反应混合物冷却至室温后加水, 析出的 沉淀经抽滤、干燥得到酰化产物粗产物, 不经提纯, 直 接常温水解, 经分离提纯得到目标产物 3-乙酰基-1-甲 基-2-羟基-5-卤素吲哚 $(\mathbf{6 a} \sim \mathbf{6 0})$, 合成路线见 Scheme 3 .

为了证实化合物 $\mathbf{6 a}$ 的结构, 对其进行了 $I R,{ }^{1} \mathrm{H}$ $\mathrm{NMR},{ }^{13} \mathrm{C} N \mathrm{NMR}$ 等光谱测定和元素分析. 在 $6 \mathbf{a}$ 的 IR 图 中我们发现, $3072 \mathrm{~cm}^{-1}$ 处出现强吸收峰为 $\mathrm{OH}$ 的伸缩振 


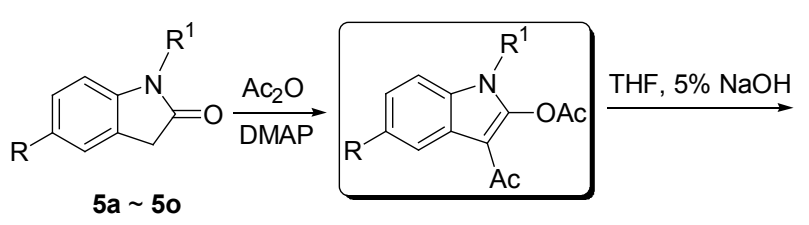<smiles>[R]c1ccc2c(c1)c(C#N)c(O)n2[R]</smiles>

$6 a \sim 60$

$\mathrm{R}=\mathrm{F}, \mathrm{Cl}, \mathrm{Br}$

$\mathrm{R}^{1}=\mathrm{Me}, \mathrm{Et}, n-\mathrm{Bu}, \mathrm{Bn}, 4-\mathrm{ClC}_{6} \mathrm{H}_{4} \mathrm{CH}_{2}$

Scheme 3

动, $2935 \mathrm{~cm}^{-1}$ 处出现的强吸收峰为双键的 $\mathrm{C}=\mathrm{C}$ 的特征 吸收峰, $1663 \mathrm{~cm}^{-1}$ 处出现的强吸收峰, 为 $\mathrm{C}=\mathrm{O}$ 的伸缩 振动. ${ }^{1} \mathrm{H}$ NMR 中, $\delta 13.68$ 处出现的多单峰归属于羟基上 的质子氢, $\delta 7.00$ 处出现的双重峰 $(J=9.0 \mathrm{~Hz})$, 归属于吲 哚环上 4 位碳上的质子氢, $\delta 6.85$ 处出现的三重峰 $(J=8.9$ $\mathrm{Hz})$, 归属于吲哚环上 7 位碳上的质子氢, $\delta 6.77$ 处出现 的三重峰 $(J=8.5 \mathrm{~Hz})$, 归属于吲哚环上 6 位碳上的质子 氢, $\delta 3.26$ 处出现的单重峰, 归属于 1 位氮上甲基上的质 子氢, $\delta 2.36$ 处出现的单重峰, 归属于 3 位乙酰基上甲基 上的质子氢. 在 ${ }^{13} \mathrm{C}$ NMR 中, $\delta 173.09$ 处出现了羰基碳 的特征吸收峰. 元素分析数据也与我们推测的化合物结 构相符. 以上数据均证实所得到的化合物 $\mathbf{6 a}$ 是 3 -乙酰 基-1-甲基-2-羊基-5-氟吲哚.

为了验证该合成方法的适用性, 三种卤素取代吲哚 酮的氮烃基化产物在 $150{ }^{\circ} \mathrm{C}$ 条件下反应 $12 \mathrm{~h}$, 其反应结 果(表 5). 从表 5 我们可以看出, 不同卤素取代吲哚酮在 该合成条件下反应，以良好的收率(32.6\% 80.5\%)得到 目标产物 6a $\sim 60$, 其结构经 ${ }^{1} \mathrm{H} N \mathrm{NMR},{ }^{13} \mathrm{C} N M R$, IR 和元 素分析得以证实. 当苯环上的卤素吸电子能力越来越强 是反应的时间相对的越来越短, 产率更高, 随着氮上烃 基的基团越来越大反应的时间越来越长，产率也越低， 但 6d 和 $6 \mathrm{e}$ 比较特殊, 这有待我们继续深入的研究和分 析.

\section{3 结论}

综上所述, 本文以卤素取代苯胺为起始原料合成靛 红, 产率均有所提高. 再经烃基化反应, 还原反应, 酰 化和水解反应得到一系列新型的 3-乙酰基-1-烃基-2-差弪 基-5-卤吲哚产物，具有反应条件温和、反应时间短、收 率良好和无毒廉价的催化剂的优点. 作为结构新颖的一 系列化合物, 将会为筛选具有更好的生理活性药物提供 更多的先导化合物.

表 5 1-取代吲哚-2-酮乙酰化的反应结果

Table 5 Results of acetylation of 5-halo-1-substituted indolin-2ones<smiles>CCN1C(=O)Cc2cc(F)ccc2N1CCS(=O)(=O)c1ccc(F)cc1</smiles>

3

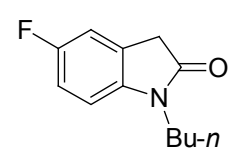<smiles>CCCCCCn1c(O)c(C(C)=O)c2cc(F)ccc21</smiles>

4<smiles>O=C1Cc2cc(F)ccc2N1Cc1ccccc1</smiles><smiles>O=C1Cc2cc(F)ccc2N1Cc1ccccc1</smiles><smiles>CN1C(=O)Cc2cc(Cl)ccc21</smiles><smiles>CCN1C(=O)Cc2cc(Cl)ccc21</smiles>

8<smiles>O=C1Cc2cc(Cl)ccc2N1Br</smiles><smiles>O=C1Cc2cc(Cl)ccc2N1Cc1ccccc1</smiles>

10<smiles>O=C1Cc2cc(Cl)ccc2N1Cc1ccccc1</smiles><smiles>CC(=O)c1c(O)n(Cc2ccccc2)c2ccc(F)cc12</smiles>
67.0 80.5 45.2 35.3 32.6 40.1 38.3 
续表<smiles>CCN1C(=O)Cc2cc(Br)ccc21</smiles>

${ }^{a}$ 分离产率.

\section{References}

[1] Jun, Q. R.; Ning, H.; Hui, X.; Liu, M. Y.; Min, L.; Yong, T. Z. Bioorg. Med. Chem. Lett. 2010, 20, 3534.

[2] Pais, G. C. G.; Zhang, X.; Marchand, C.; Neamati, N.; Cowansage, K.; Svarovskaia, E. S.; Pathak, V. K.; Tang, Y.; Nicklaus, M.; Pommier, Y.; Burke, Jr. T. R. J. Med. Chem. 2002, 45, 3184.

[3] Ferro, S.; Barreca, M. L.; Luca, L. D.; Rao, A.; Monforte, A. M.; Debyser, Z.; Witrouw, M.; Chimirri, A. Arch. Pharm. Chem. Life Sci. 2007, 340, 292.

[4] Abdel-gawad, H.; Mohamed, H. A.; Dawood, K. M.; Badria, F. A.-R. Chem. Pharm. Bull. 2010, 58, 1529.

[5] Sriniva, P.; Raghavan, S. A. V.; Jagadeeh-bababu, R.; Gupta, C. N. V. H. B.; Sridhar, N.; Veeranjaneyulu, A.; Parimoo, P. Pharmacol. Commun. 1999, 5, 95.

[6] Giraud, F.; Alves, G.; Debiton, E.; Nauton, L.; Thery, V.; Durieu, E.; Ferandin, Y.; Lozach, O.; Meijer, L.; Anizon, F.; Pereira, E.; Moreau, P. J. Med. Chem. 2011, 54, 4474.

[7] Chennamaneni, S.; Zhong, B.; Lama, R.; Su, B. Eur. J. Med. Chem. 2012, 56, 17.

[8] Bruel, A.; Logé, C.; Tauzia, M. L.; Ravache, M.; Guevel, R.; Guillouzo, C.; Lohier, J. F.; Oliveira, Santos. J. S.; Lozach, O.; Meijer, L.; Ruchaud, S.; Bénédetti, H.; Robert, J. M. Eur. J. Med. Chem. 2012, 57, 225.

[9] Martel-Frachet, V.; Kadri, M.; Boumendjel, A.; Ronot, X. Bioorg. Med. Chem. 2011, 19, 6143.

[10] Kumar, D.; Kumar, N. M.; Sundaree, S.; Johnson, E. O.; Shah, K.
Eur. J. Med. Chem. 2010, 45, 1244

[11] Subba Reddy, B. V. S.; Rajeswari, N.; Sarangapani, M.; Reddy, G. R.; Msdan, C.; Kumar, K. P.; Rao, M. S. Bioorg. Med. Chem. Lett. 2011, 21, 6510 .

[12] Subba, R. B. V.; Rajeswari, N.; Sarangapani, M.; Prashanthi, Y.; Ganji, R. J.; Addlagatta, A. Bioorg. Med. Chem. Lett. 2012, 22, 2460.

[13] Jørgensen, M.; Jørgensen, P. N.; Christoffersen, C. T.; Jensen, K. G.; Balle, T.; Bang-Andersen, B. Bioorg. Med. Chem. 2013, 21, 196.

[14] Shen, X. Q.; Wu Y. L.; Qian, H. J. Chem. Ind. Times 2012, 26, 29 (in Chinese).

(沈学全, 吴金龙, 钱海均, 化工时刊, 2012, 26, 29.)

[15] Zhang, X. F.; Liu, H. Y.; Gao, W. T. J. Bohai Univ. (Nat. Sci.) 2009, 30, 212 (in Chinese).

(张晓飞, 刘华业, 高文涛, 渤海大学学报 (自然科学版), 2009, 30, 212.)

[16] Singh, R. P.; Majumder, U.; Shreeve, J. M. J. Org. Chem. 2001, 19 , 6263.

[17] Lei, J.; Fang, Q.; Yuan, M.-S.; Liu, Z.-Q.; Shen, Y.-X.; Chen, H.-F. Org. Lett. 2010, 22, 5192.

[18] Hennessy, E. J.; Buchwald, S. L. J. Am. Chem. Soc. 2003, 40, 12084.

[19] Prandi, C.; Occhiato, E. G.; Tabasso, S.; Bonfante, P.; Scarpi, D.; Bova, M. E.; Miletto, I. Eur. J. Org. Chem. 2011, 20, 3781.

[20] Hamaue, N.; Mimami, M.; Terado, M.; Hirafuji, M.; Endo, T.; Machida, M.; Hiroshige, T.; Ogata, A.; Tashiro, K.; Saito, H.; Parrez, S. Neurotoxicology 2004. 25, 205.

[21] Igosheva, N.; Lorz, C.; Conner, E. Neurochem. Int. 2005, 47, 216.

[22] Minami, M.; Hamaue, N.; Hirafuji, M.; Saito, H.; Hiroshige, T.; Ogata, A.; Tashiro, K.; Parvez, S. H. J. Neural Transm. Suppl. 2006, 71,87 .

[23] Aulabaugh, A.; Kapoor, B.; Huang, X. Y.; Dollings, P.; Hum, W. T.; Banker, A.; Wood, A.; Ellestad, G. Biochemistry 2007, 46, 9462.

[24] Ogata, A.; Hamaue, N.; Terado, M.; Mimami, M.; Nagashima, K.; Tashiro, K. J. Neurol. Sci. 2003, 206, 79.

[25] Kenner, C.; Rice, B. J.; Boone, A. B.; Rubin, T. J. J. Med. Chem. 1976, 19, 887.

[26] Welstead, Jr.; W. J.; Moran, H. W.; Stauffer, H. F.; Turnbull, L. B.; Sancilio, L. F. J. Med. Chem. 1979, 22, 1074.

[27] Rivalle, C.; Bisagni, E. J. Heterocycl. Chem. 1997, 34, 441.

[28] Gassman, P. G.; Cue Jr. B. W.; Luh, T. Y. J. Org. Chem. 1977, 42, 1344.

[29] Lackey, K.; Besterman, J. M.; Fletcher, W.; Leitner, P.; Morton, B.; Sternbach, D. D. J. Med. Chem. 1995, 38, 906

[30] Garden, S. J.; Torees, J.; Ferrira, A. A.; Silva, R. B.; Pinto, A. C. Tetrahedron Lett. 1997, 38, 1501.

[31] Kawaguchi, H.; Mizuta, Y.; Sugai, F.; Saito, S. EP 19950308606, 1996 [Chem. Abstr. 1996, 125, 181167].

[32] Friedman, S. J. US 3659011, 1972 [Chem. Abstr. 1972, 77, 125053].

[33] Marti, C.; Carreira, E. M. J. Am. Chem. Soc. 127, 11505.

[34] Cui, X. J.; Shi, F.; Zhang, Y. Tetrahedron Lett. 2010, 51, 2048.

[35] Matos, I.; Prez-Mayora, E.; Soriano, E.; Zukal, A.; Martín-Aranda R. M.; López-Peinado, A. J.; Fonseca, I.; Čejka, J. Chem. Eng. J. 2010, 161, 377.

[36] Reddy, C. R.; Jithender, E. Tetrahedron Lett. 2009, 50, 5633.

[37] Shah, H. C.; Shah, V. H.; Desai, N. D. ChemInform 2010, 40, 540.

[38] Jha, M.; Chou, T. Y.; Blunt, B. Tetrahedron 2011, 67, 982.

(Cheng, F.) 